NBSIR 82-2565

\title{
Profiles of Computer Programmers in the Executive Branch of the Federal Government
}

U.S. DEPARTMENT OF COMMERCE National Bureau of Standards Institute for Computer Sciences and Technology Center for Programming Sciences and Technology Washington, DC 20234

September 1982

Issued March 1983

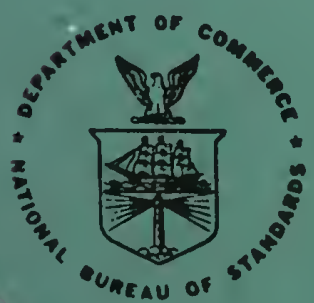

U.S. DEPARTMENT OF COMMERCE NATIONAL BUREAU OF STANDARDS

\section{FILE COPY DO NOT TAKE}



NBSIR $82-2565$

PROFILES OF COMPUTER PROGRAMMERS

IN THE EXECUTIVE BRANCH OF THE

FEDERAL GOVERNMENT

Patricia B. Powell, Editor

U.S. DEPARTMENT OF COMMERCE

National Bureau of Standards

Institute for Computer Sciences and Technology

Center for Programming Sciences and Technology

Washington, DC 20234

September 1982

Issued March 1983

U.S. DEPARTMENT OF COMMERCE, Malcolm Baldrigo, Secrotary NATIONAL BUREAU OF STANDARDS. Emest Ambler, Director 



\title{
PROFILES OF COMPUTER PROGRAMMERS
}

\author{
IN THE
}

\section{EXECUTIVE BRANCH OF THE FEDERAL GOVERNMENT}

Patricia B. Powell, Editor

september, 1982

Center for Programming Sciences and Technology Institute for Computer Sciences and Technology National Bureau of Standards

Washington, D.C. 20234 

TABLE OF CONTENTS

II Observations 1

II Organization of Data 2

IV Selection of Organizations for Study 3

$\mathrm{V}$ Summary of Descriptive Data of Selected 3

Computer Organizations

VI Summary of Programmer Profile Data 5

$\begin{array}{ll}\text { VII Organization A } & 7\end{array}$

VIII Organization B $r$

IX Organization C $\quad 14$

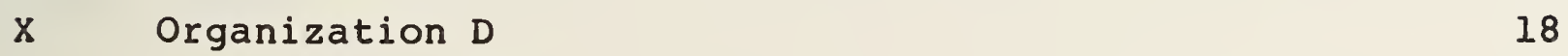

$\begin{array}{lll}X I & \text { Organization E } & 21\end{array}$

$\begin{array}{lr}\text { XII Organization F } & 25\end{array}$

$\begin{array}{lr}\text { XII Organization G } & 28\end{array}$

$\begin{array}{ll}\text { XIV Organization } \mathrm{H} & 31\end{array}$

Chart 1 Tabulations of Percentages from Computer Programmer Profile statistics

Appendix 1 Statistics from the General Personnel Data File of the office of Personnel Management

Table 1 Number and Percentage of Computer Specialists by Age, Grade, and Education

Table 2 Number and Percentage of Computer Specialists by Age in Those Agencies Employing Over 28 of Total Number

Table 3 Number and Percentage of Computer specialists by Grade in Those Agencies Employing Over 28 of Total Number

Table 4 Number and Percentage of Computer specialists by Education in Those Agencies Employing Over $2 \%$ of Total Number 
Table 5 Number and Percent of Computer Specialists by Agency

Table 6 Number of Computer specialists by Age Groupings for Agencies and Sub-agencies 10

Table 7 Number of Computer Specialists by Grade Levels for Agencies and Sub-agencies

Table 8 Number of Computer specialists by Educational Levels for Agencies and Sub-agencies

Appendix 2 Computer Programmer Survey - Individual Programmer Profile

Appendix 3 Computer Programmer Survey - Description of Activity of Organization 
This report is a detailed programmer survey compiled from interviews with eight selected organizations and an OPM data base. The survey includes staffing, hardware, programming activities and languages, contract support, programmer recruiting, quality control, personnel profile, and programmer activities. The OPM data base is summarized by age, grade, and education for the computer specialists job series in the Washington Metropolitan Area. Observations from the data received indicate that technical information should be geared towards individuals with substantial practical experience and a high school education augmented by technical trainingi a very high percent of the programming in the selected organizations is done in COBOL; more effective tools are needed to assist in software quality; the emphases needed in the production of standards and technical guidance are practicality and simplicity.

KEYWORDS: computer programmers; computer specialists; Federal civilian organizations; OPM data base; profile of computer programmers

\section{ACKNOWLEDGEMENTS}

This report was funded by the National Bureau of Standards' Institute for Computer Sciences and Technology under U.S. Department of Commerce purchase order NB800NAAG4467. The author is Ralph A. Simmons. The views and conclusions expressed are those of the contractor and do not necessarily represent the official policies of the Department of Commerce or the United States Government.

The document has been edited for publication. Comments or questions should be directed to:

Systems and Software Technology Division

Room B266, Building 225

National Bureau of Standards

Washington, D.C. 20234 

Page 1

I. Introduction

The Institute for Computer Sciences and Technology (ICST) carries out the following responsibilities under P.L. 89-306 (Brooks Act) to improve the Federal government's management and use of ADP:

- develops Federal automatic data processing standards;

- provides agencies with scientific and technological advisory services relating to ADP;

- undertakes necessary research in computer science and technology.

In partial fulfillment of Brooks Act responsibilities, ICST issues Internal Reports (IR). The purpose of this report is to present descriptive data of selected computer organizations in the Federal Government and their programming personnel to assist the staff of the Institute for Computer Sciences and Technology in the development of programming standards and in the preparation of technical guidance.

\section{Observations}

The requirements for this report do not include conclusions or recommendations. However, the following observations are believed to be of particular importance to this purpose and are, therefore, highlighted here:

A. The preparation of technial information intended for use by programmers/analysts in the Federal Government should be geared for individuals with substantial practical experience but not so complex that it cannot be effectively used by someone with only a high school level formal education. However, the average user can be expected to have had the high school education augmented by technical training. These potential customers are, more likely, GS-12's or higher and over 30 years of age. Probably a great many will be over 40 .

B. A very high percentage of programming in the selected organizations is being done in COBOL and in support of what are characterized as business-type applications. The majority of this work is either for the development of new systems or for the maintenance of existing systems.

C. When software quality was discussed with two of the managers, FIPS PUBS were mentioned as being 
valuable standards. The FIPS PUBS 31 and 64 were singled out as being particularly good while FIPS PUB 38 was termed useful but in need of updating. One manager labeled FIPS PUB 49 as inadequate. More effective tools are needed to assist in software quality and special mention was made of the need for expert guidance in computer performance management.

D. The emphases needed in the production of standards and technical guidance are practicality and simplicity.

III. Organization of the Data

A. The report is supported by data collected during the Spring of 1981 from the following:

1. Individual Programmer Profile

A copy of this survey form is included as Appendix 2. Its purpose was to expedite the collection of data describing certain characteristics of Federal computer programmers in the 334 series (Computer specialists) and their jobs. A copy of this form was completed by each programmer surveyed.

2. Description of Activity of Organization

A copy of this survey form is included as Appendix 3. Its purpose was to expedite the collection of data describing the individual organization for which the programmers work and the more pertinent activities of that organization. The survey form was reviewed during interviews with managers of the selected organizations and was subsequently completed by them.

B. The data collected through the survey forms were tabulated, organized and reviewed. Summaries of the results for each selected organization are included in this report and consist of three major parts: organization description, individual programmer profiles and tabulation of programmer profile data. Additional statistical summaries from OPM data, September, 1980, are included as Appendices and have been prepared to assist in the interpretation of the data. Only limited analysis is included in this report because the main objective is to present the data for direct interpretation. 
IV. Selection of Organizations for study

The following criteria for organizational selection were:

1. Civilian organizations in the Executive Branch of the Federal Government;

2. Located in the Washington Metropolitan area as defined by the standard Metropolitan Statistical Area(SMSA) as defined by the Bureau of the Census (District of Columbia; Arlington, Fairfax, Loudon, and Prince William counties and Alexandria, Fairfax, Manassas, Manassas Park and Falls Church cities in Virginia; and Charles, Montgomery and Prince Georges counties in Maryland.); and

3. Ten or more computer programmers on the staff.

Within the surveyed organizations, participants were selected to provide a representative sample of typical functions and personnel with particular attention paid to:

1. Type of programming

2. Size of programming staff

3. Type of applications

4. Levels of education, GS range and age span of the programming staff

v. Summary of Descriptive Data of Selected Computer Organizations

A. Staff

Of the eight organizations surveyed, five have staffs with total strengths of 60-70 people; three have total strengths of 40-50 people.

B. Hardware

In each case, the mainframe hardware used is that of the respective agency-level headquarters. The equipment listed as in-house consists of remote terminals and printers. In four of the organizations, 
over two-thirds of programmer input is submitted through interactive terminals.

C. Programming activities

The managers of four organizations have characterized the programing as in support of business-type applications. In each case, almost one-half of the programming is for new development, with maintenance an important second activity. The other four organizations support statistical, graphics, and computer systems functions.

D. Programming languages

In four of the organizations, COBOL is the principal language used. In fact, it is used for over 908 of the work. FORTRAN, ALGOL, PL-l and EASYTRIEVE were also mentioned. One of the organizations uses Assembler as the principal language; two use FORTRAN principally; one uses COBOL or FORTRAN depending on the application.

E. Contract support

The levels of contract assistance reported were low in each case. Practically none of the programming is accomplished through contract.

F. Programmer recruiting

The principal sources for recruitment are college recruitments and the office of Personnel Management system PACE and the supporting registers. One organization reported no recruiting. It is particularly interesting to note that organization $B$ concentrates its recruitment at the GS-5 level and looks primarily for applicants with aptitude rather than experience. This is reflected in its higher percentage of lower graded employees $(308$ at the 5-7 level) than in the other organizations. The manager at Organization $C$ made the observation that it was difficult to find mid and senior-level programmers, and that it was almost impossible to find programmers with experience with Burroughs hardware as opposed to IBM hardware. Several organizations reported recruiting difficulties at Universities due to severe competition from private industry and the lower pay scale of the Federal Government.

G. Qualiy control

six of the eight organizations reported the existence 
of programs for quality control. They reported the development and use of internal standards and guide lines as well as the use of FIPS PUBS. See the individual organizational summaries for details. It is interesting to note that organizations $D$ and $G$ reported a comprehensive procedure for quality assurance but listed only FIPS PUBS and an editor as specific means of accomplishing quality assurance. There appears to be a misconception about the meaning of quality assurance.

\section{Summary of Programmer Profile Data}

A. The detailed statistics for each organization are given with the individual organization. Comparative percentages (Chart 1 ) are summarized following the reports on the individual organizations. The statistics used for the office of Personnel Management on the charts are fron the Central Personnel Data File of OPM, September, 1980. These OPM statistics are for all agencies of the Executive Branch (excluding the military departments) in the Washington Metropolitan area. They are also for the entire 334 series (computer specialists) which includes analysts as well as programmers.

B. The following observations are made in reference to the charts:

Age Groupings

In general, the staffs have fewer people in the 30-39 and 40 and over age groupings than the OPM statistics show. However, three of the eight staffs are close to the OPM percentage for the 40 and over age group: for the $30-39$ age group, three organizations are near the OPM percentage.

Grade Groupings

The grade structure in the groups surveyed does not match the OPM statistics. There are generally less staff members in grades 13 and over and more in grades 9 through 12 .

Time-in-agency Groupings

Half of the organizations have the largest percentage in the 3-8 year category. The other half have the largest percentage in the 1-3 year category. No data on time-in-agency was extracted from the OPM file. 


\section{Levels of Education}

Two of the eight organizations conform to the OPM statistics which indicate that about one-half of the programmers have not received a bachelor's degree. The remaining six organizations have a higher percentage of personnel who have earned a bachelor's degree. For the master's degree, four of the organizations are under the OPM percentage of 98 and four are over the OPM percentage. There is one programmer with a doctorate in the eight organizations and only $1 \%$ of the total computer specialists in the OPM file have attained that level.

The following eight sections contain the detailed statistics for each organization surveyed. It should be noted that the information presented for an organization under general description may differ from that presented under the individual profiles as different people completed the data collection forms. 
VII. Organization A

A. Summary of Organization Description

1. Staff: Office of Director 5

Application Systems $\quad 10$

Financial systems $\quad 11$

Management Systems $\quad 17$

Special Applic. Systems 14

Total

57

2. Hardware: Large mainframe

Internally has intelligent terminals and low-speed terminals.

3. Programming activities:

Approximately $4 \varnothing$ systems analysts/computer specialists programmers do both analysis and programming of primarily COBOL systems using batch up-date through intelligent terminals with output both batch and interactive. All programming is in support of business-type applications. About one-half of the programming is for new applications and one-half for maintenance.

4. Programming languages:

Principal: COBOL

Other: Fortran and EASYTRIEVE

5. Contract support:

a. Level of effort: 6 man-years/yr. $\$ 350, \emptyset \emptyset \sigma / \mathrm{yr}$.

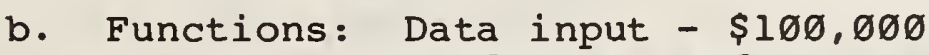

Hardware \& software support - $\$ 250,000$ (to be phased out)

6. Programmer recruiting:

Source: Personal reference

7. Quality control: No data submitted 
B. Summary of Individual Programmer Profiles

1. Total number surveyed: 23

2. Age summary:

$\begin{array}{rr}\text { No. } & 8 \\ 9 & 4 \emptyset \\ 12 & 52 \\ 1 & 4 \\ 1 & 4\end{array}$

3. Grade summary:

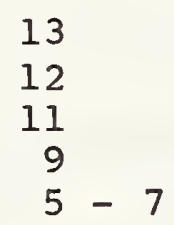

$\begin{array}{rr}10 & 43 \\ 5 & 22 \\ 5 & 22 \\ 1 & 4 \\ 2 & 9\end{array}$

4. Time-in-agency summary:

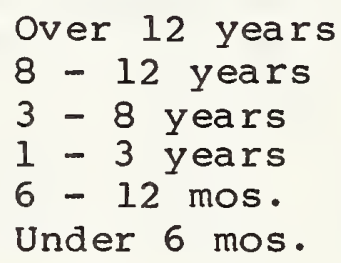

$\begin{array}{rr}1 & 4 \\ 5 & 22 \\ 9 & 40 \\ 6 & 26 \\ 1 & 4 \\ 1 & 4\end{array}$

5. Education summary:

$\begin{array}{lrr}\text { Master's degree } & 1 & 4 \\ \text { Bachelor's deg. } & 10 & 44 \\ \text { High school grad. } & 12 & 52\end{array}$

6. Computer system used: large mainframe for 1098 of time

7. Average reported input method for programming:

Batch: $48 \%$

Interactive: $52 \%$

8. Type of programming activity: (order of area in which most time is reportedly spent)

Code

Test

Design

Maintenance

Documentation 
9. Average reported time spent by phase of application programming:

$\begin{array}{lr}\text { New development } & 45 \% \\ \text { Conversion } & 16 \% \\ \text { New versions of old programs } & 14 \% \\ \text { Maintenance } & 27 \% \\ \text { Miscellaneous } & 3 \%\end{array}$

C. Tabulation of Programmer Profile Data

\begin{tabular}{|c|c|c|c|c|c|}
\hline & ge & Grade & Time-in- & agency & Education \\
\hline & $\theta+$ & 13 & $8-12$ & yrs. & B \\
\hline & $\theta+$ & 13 & $3-8$ & yrs. & B \\
\hline & $\theta+$ & 13 & $3-8$ & yrs. & B \\
\hline & $\theta+$ & 13 & $3-8$ & yrs. & HS \\
\hline & $\theta+$ & $\begin{array}{l}13 \\
13\end{array}$ & $\begin{array}{ll}1- & 3 \\
\text { Less } & \text { th }\end{array}$ & $\begin{array}{l}\text { yrs. } \\
\text { an } 6 \text { mos. }\end{array}$ & $\begin{array}{r}\text { M } \\
\text { HS }\end{array}$ \\
\hline & $\theta+$ & 12 & $8-12$ & yrs. & HS \\
\hline & $\theta+$ & 11 & Over 1 & 2 yrs. & B \\
\hline & $x+$ & 11 & $1-3$ & yrs. & B \\
\hline 30 & -39 & 13 & $8-12$ & yrs. & B \\
\hline 30 & -39 & 13 & $3-8$ & yrs. & HS \\
\hline 39 & -39 & 13 & $3-8$ & yrs. & HS \\
\hline 30 & -39 & 13 & $1-3$ & yrs. & B \\
\hline 30 & -.39 & 12 & $3-8$ & yrs. & HS \\
\hline 30 & $-\quad 39$ & 12 & $6-12$ & mos. & B \\
\hline 30 & -39 & 12 & $8-12$ & yrs. & B \\
\hline 39 & -39 & 11 & $3-8$ & yrs. & HS \\
\hline 30 & -39 & 11 & $1-$ & yrs. & HS \\
\hline 30 & -39 & 9 & $3-8$ & yrs. & HS \\
\hline 30 & -39 & $5-7$ & $1-3$ & yrs. & B \\
\hline 30 & -39 & $5-7$ & $1-$ & - yrs. & HS \\
\hline 25 & -29 & 12 & $8-12$ & yrs. & HS \\
\hline 20 & -24 & 11 & $3-8$ & yrs. & HS \\
\hline
\end{tabular}

Note: Codes for education: M Master's Degree B Bachelor's Degree HS High School Graduate 


\section{Organization B}

A. Summary of Organization Description

1. Staff: Office of Chief 3

Planning \& Standards 5

Program Systems 22

Administrative Systems $\quad \underline{24}$

Total 54

2. Hardware: Large Mainframe

3. Programming activities:

Staff does all phases of systems analysis, design and programming. Majority of programming is in support of business-type applications. About $45 \%$ of programming is for new development, $45 \%$ for maintenance and $10 \%$ for conversion.

4. Programming languages:

Principal: COBOL (95\%)

Other: PL-1 and FORTRAN

5. Contract support:

a. Level of effort: $5 \%$ of programming

b. Functions: Programming

6. Programmer recruiting:

a. Sources: PACE - 998; Other - 198

b. Means of contact: OPM register \& public announcement

c. Special programs: Upward mobility \& student work program

d. Comments: Hire at Gs-5 level \& look for applicants with aptitude as opposed to experience.

7. Quality control:

a. Standards used: FIPS PUB. 38 Level III 
b. Guidelines used:

structured programming techniques \& structured design techniques, internal guidelines

c. Tools used:

test data generation, statistical analysis, management of files and data, documentation and reporting

d. Comments :

Comprehensive procedures are used for quality control, assurance and testing. Extensive in-house and outside training in "best" methods's provided. Guidance in additional language standards is needed.

B. Summary of Individual Programmer Profiles

1. Total number surveyed: 36

2. Age summary:

$\begin{array}{rl}\text { No. } & 8 \\ 5 & 14 \\ 15 & 42 \\ 13 & 36 \\ 3 & 8\end{array}$

3. Grade summary:

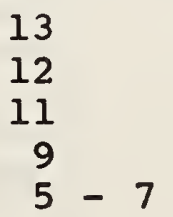

$\begin{array}{rr}3 & 8 \\ 15 & 42 \\ 5 & 14 \\ 2 & 6 \\ 11 & 30\end{array}$

4. Time-in-agency

$$
\begin{aligned}
& \text { Over } 12 \text { years } \\
& 8-12 \text { yrs. } \\
& 3-8 \text { yrs. } \\
& 1-3 \text { yrs. } \\
& 6-12 \text { mos. } \\
& \text { Under } 6 \text { mos. }
\end{aligned}
$$

$\begin{array}{rr}3 & 8 \\ 6 & 17 \\ 14 & 39 \\ 12 & 33 \\ 0 & 0 \\ 1 & 3\end{array}$

5. Education summary:

$\begin{array}{lrr}\text { Master's Degree } & 1 & 3 \\ \text { Bachelor's Degree } & 31 & 86 \\ \text { High school grad. } & 4 & 11\end{array}$


6. Computer system used: Large Mainframe

7. Average reported input method for programming:

Batch: $73 \%$
Interactive: $27 \%$

8. Type of programming activity: (order of area in which most time is reportedly spent)

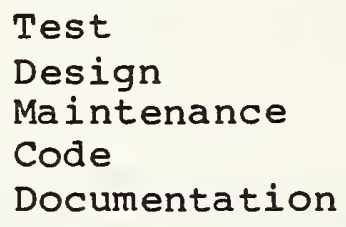

9. Average reported time spent by phase of application programming:

$\begin{array}{lr}\text { New development } & 33 \% \\ \text { Conversion } & 15 \% \\ \text { New versions of old programs } & 17 \% \\ \text { Maintenance } & 33 \% \\ \text { Other } & 2 \%\end{array}$

C. Tabulation of Programmer Profile Data

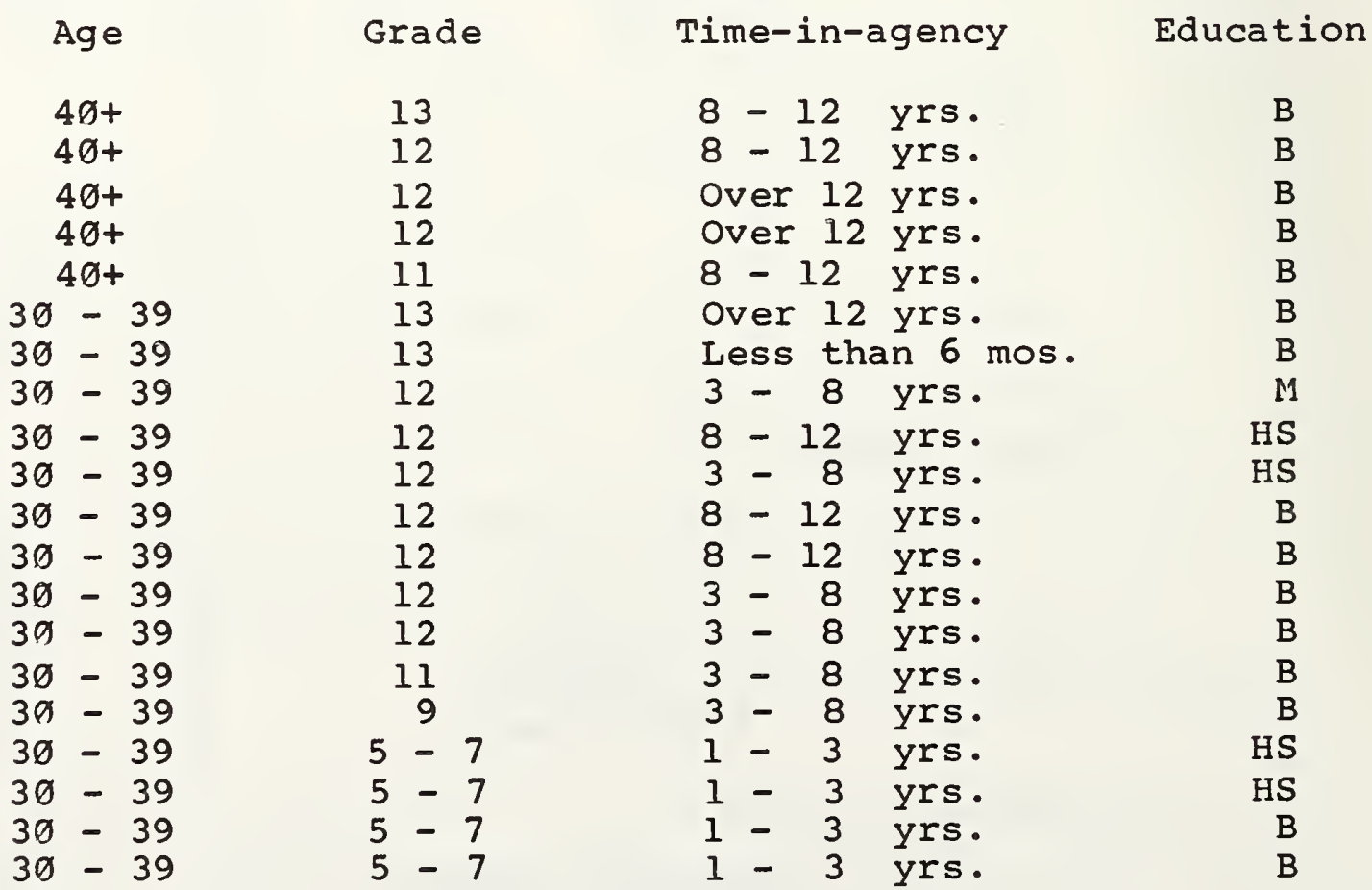


C. Tabulation of Programmer Profile Data (continued)

\begin{tabular}{|c|c|c|c|c|c|c|}
\hline \multicolumn{2}{|c|}{ Age } & Grade & \multicolumn{3}{|c|}{ Time-in-agency } & Education \\
\hline 25 & -29 & 12 & $3-$ & 8 & yrs. & B \\
\hline 25 & -29 & 12 & $3-$ & 8 & yrs. & B \\
\hline 25 & -29 & 12 & $3-$ & 8 & yrs. & B \\
\hline 25 & -29 & 12 & $3-$ & 8 & yrs. & B \\
\hline 25 & -29 & 12 & $3-$ & 8 & yrs. & B \\
\hline 25 & -29 & 11 & $3-$ & 8 & yrs. & B \\
\hline 25 & -29 & 11 & $3-$ & 8 & yrs. & B \\
\hline 25 & -29 & 11 & $3-$ & 8 & yrs. & B \\
\hline 25 & -29 & $5-7$ & $1-$ & 3 & yrs. & B \\
\hline 25 & -29 & $5-7$ & $1-$ & 3 & yrs. & B \\
\hline 25 & -29 & $5-7$ & $1-$ & 3 & yrs. & B \\
\hline 25 & -29 & $5-7$ & $1-$ & 3 & yrs. & B \\
\hline 25 & -29 & $5-7$ & $1-$ & 3 & yrs. & B \\
\hline $2 \varnothing$ & -24 & 9 & $1-$ & 3 & yrs. & B \\
\hline 29 & -24 & $5-7$ & $1-$ & 3 & yrs. & B \\
\hline $2 \varnothing$ & -24 & $5-7$ & $1-$ & 3 & yrs. & B \\
\hline
\end{tabular}

\footnotetext{
Notes: Codes for education: M Master's Degree

B Bachelor's Degree

HS High school graduate
}

All but three programmers listed specialized computer education, however, only one majored in computer science. 
IX. Organization C

A. Summary of Organization Description

1. Staff: Software Development 37

Production Management 14

Quality Assurance $\underline{9}$

Total 60

2. Hardware: Large Mainframe

3. Programming activities:

Staff of 37 involved in programming. Responsible for maintenance and enhancement of large (about 600 programs) logistics system. Majority of programming is in support of business-type applications. About $50 \%$ of programming is for new development, $20 \%$ for conversion, $20 \%$ for maintenance and $10 \%$ for other.

4. Programming languages:

Principal: COBOL (94\%)

Other: FORTRAN (18); ALGOL (58)

5. Contract support:

About $\$ 5 \varnothing, \emptyset \emptyset \emptyset$ per year spent for consulting services. No actual programming done under contract.

6. Programmer recruiting:

a. Sources: In-house (trainees)

OPM (PACE \& registers)

Word-of-mouth

b. Special Programs: Career intern development system

c. Comments: Easy to recruit trainees; difficult to find mid to senior level programmers; not so hard to find IBM experience; impossible for ALGOL experience; now a sellers market. 
7. Quality control:

a. Standards used: FIPS PUBS $38 \& 64$

Internally developed standards

b. Tools used: Documentation generator

c. Comments :

Used procedures for quality assurance and testing. A new Quality Assurance Organization is currently being established. Some training, both in-house \& outside, in "best" methods is provided.

d. Quality of standards and needs:

FIPS PUB 38 is viewed as in need of updating while FIPS PUB 64 is considered excellent and will be incorporated intact as the agency internal standard. FIPS PUB 31 is thought to be good but FIPS PUB 49 is considered inadequate. There is a need for expert guidance in computer performance management.

B. Summary of Individual Programmer Profiles

1. Total number surveyed: 33

2. Age summary:

No. $\quad$ of

$$
\begin{aligned}
& 40 \text { and over } \\
& 30-39 \\
& 25-29
\end{aligned}
$$

3. Grade summary:

Over 13
13
12
11
9
$5-7$

$\begin{array}{rr}3 & 9 \\ 6 & 19 \\ 15 & 45 \\ 2 & 6 \\ 7 & 21 \\ 0 & 0\end{array}$

4. Time-in-agency summary:

$$
\begin{aligned}
& \text { Over } 12 \text { years } \\
& 8-12 \text { years } \\
& 3-8 \text { years } \\
& 1-3 \text { years } \\
& 6-12 \text { mos. } \\
& \text { Under } 6 \text { mos. }
\end{aligned}
$$

$\begin{array}{rr}6 & 18 \\ 8 & 24 \\ 14 & 43 \\ 3 & 9 \\ 1 & 3 \\ 1 & 3\end{array}$


5. Education summary:

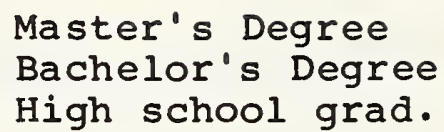

No.

\%

$\begin{array}{rr}2 & 6 \\ 12 & 36 \\ 19 & 58\end{array}$

(four have an associate degree in ADP and two have degrees in $\mathrm{ADP}$.)

6. Computer system used: large mainframe

7. Average reported input method for programming:

Batch: $30 \%$

Interactive: $70 \%$

8. Type of programming activity: (order of area in which most time is reportedly spent)

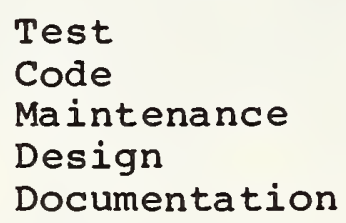

9. Average reported time spent by phase of application programming:

$\begin{array}{lr}\text { New development } & 46 \% \\ \text { Conversion } & 11 \% \\ \text { New versions of old programs } & 11 \% \\ \text { Maintenance } & 29 \% \\ \text { Other } & 3 \%\end{array}$

C. Tabulation of Programmer Profile Data

$\begin{array}{lc}\text { Age } & \text { Grade } \\ 4 \emptyset+ & \text { Over } 13 \\ 4 \emptyset+ & 13 \\ 4 \emptyset+ & 13 \\ 4 \emptyset+ & \text { Over } 13 \\ 4 \emptyset+ & 13 \\ 4 \emptyset+ & 13 \\ 4 \emptyset+ & 13 \\ 4 \emptyset+ & 12 \\ 4 \emptyset+ & 12 \\ 4 \emptyset+ & 12 \\ 4 \emptyset+ & 12 \\ 4 \emptyset+ & 12 \\ 4 \emptyset+ & 12 \\ 4 \emptyset+ & 9\end{array}$

Time-in-agency

Over 12 yrs.
Over 12 yrs.
Over 12 yrs.
$3-8$ yrs.
$3-8$ yrs.
$8-12$ yrs.
$8-12$ yrs.
$3-8$ yrs.
Over 12 yrs.
$8-12$ yrs.
$8-12$ yrs.
Over 12 yrs.
$8-12$ yrs.
$1-3$ yrs.

Education

HS

HS

HS

B

B

HS

HS

B

HS

$B$ 
C. Tabulation of Programmer Profile Data (continued)

$\begin{array}{cc}\text { Age } & \text { Grade } \\ 3 \emptyset-39 & \text { Over } 13 \\ 3 \emptyset-39 & 13 \\ 3 \emptyset-39 & 12 \\ 3 \emptyset-39 & 12 \\ 3 \emptyset-39 & 12 \\ 3 \emptyset-39 & 12 \\ 3 \emptyset-39 & 12 \\ 3 \emptyset-39 & 12 \\ 3 \emptyset-39 & 11 \\ 3 \emptyset-39 & 11 \\ 3 \emptyset-39 & 9 \\ 3 \emptyset-39 & 9 \\ 3 \emptyset-39 & 9 \\ 3 \emptyset-39 & 9 \\ 39-39 & 9 \\ 25-29 & 12 \\ 25-29 & 12 \\ 25-29 & 12 \\ 25-29 & 9\end{array}$

Time-in-agency

3 - 8 yrs.

8 - 12 yrs.

3 - 8 yrs.

3 - 8 yrs.

Over 12 yrs.

8 - 12 yrs.

3 - 8 yrs.

8 - 12 yrs.

3 - 8 yrs.

3 - 8 yrs.

3 - 8 yrs.

Less than 6 mos.

3 - 8 yrs.

3 - 8 yrs.

1 - 3 yrs.

6 - 12 mos.

3 - 8 yrs.

3 - 8 yrs.

1 - 3 yrs.
Education

M

B

HS

HS

B

HS

B

HS

HS

HS

B

HS

B

HS

B

B

B

HS

Note: Four of the programmers with only a high school degree have an associate degree in ADP. 
A. Summary of Organization Description

1. Staff: five branches with total of 20 computer specialists

2. Hardware: large mainframe

small mainframe

3. Programming activities:

20 computer analysts/programmers responsible for administrative systems, e.g. payroll, inventories, accounting, budget and management information systems.

4. Programming languages:

Principa 1: COBOL (998)

Other: FORTRAN and EASYTRIEVE

5. Contractor support: None

6. Programmer recruiting:

Primarily from colleges and by transfers from other government agencies.

7. Quality Control:
a. Standards used: ASCII character set COBOL
b. Guidelines used: None
c. Tools used: text editor
d. Comments:

A comprehensive procedure for quality assurance is available. 
B. Summary of Individual Programmer Profiles

1. Total number surveyed: 18

2. Age summary:

$\begin{array}{ll}\text { No. } & 8 \\ 5 & 27 \\ 3 & 17 \\ 7 & 39 \\ 3 & 17\end{array}$

3. Grade summary:

$$
\begin{aligned}
& 4 \emptyset \text { and over } \\
& 3 \emptyset-39 \\
& 25-29 \\
& 2 \emptyset-24
\end{aligned}
$$

\section{8}


9. Average reported time spent by phase of application programming:

$\begin{array}{lr}\text { New development } & 41 \% \\ \text { Conversion } & 6 \% \\ \text { New versions of old programs } & 198 \\ \text { Maintenance } & 28 \% \\ \text { Other } & 6 \%\end{array}$

C. Tabulation of Programmer Profile Data

\begin{tabular}{|c|c|c|c|}
\hline Age & Grade & Time-in-agency & Education \\
\hline $4 \emptyset+$ & 13 & Over 12 yrs. & B \\
\hline $4 \varnothing+$ & 13 & Over 12 yrs. & HS \\
\hline $40+$ & 13 & Over 12 yrs. & HS \\
\hline $49+$ & 12 & 3 - 8 yrs. & HS \\
\hline $4 \varnothing+$ & 12 & Over 12 yrs. & B \\
\hline $30-39$ & 13 & $8-12$ yrs. & B \\
\hline $30-39$ & 9 & $1-3$ yrs. & B \\
\hline $3 \emptyset-39$ & $5-7$ & 1 - 3 yrs. & HS \\
\hline $25-29$ & 12 & 3 - 8 yrs. & B \\
\hline $25-29$ & 12 & 3 - 8 yrs. & B \\
\hline $25-29$ & 12 & 3 - 8 yrs. & B \\
\hline $25-29$ & 11 & $1-3$ yrs. & B \\
\hline $25-29$ & 11 & $1-3$ yrs. & B \\
\hline $25-29$ & 11 & $1-3$ yrs. & B \\
\hline $25-29$ & $5-7$ & $1-3$ yrs. & HS \\
\hline $2 \varnothing-24$ & 9 & $1-3$ yrs. & B \\
\hline $2 \emptyset-24$ & 9 & 1 - 3 yrs. & B \\
\hline $20-24$ & $5-7$ & $1-3$ yrs. & B \\
\hline
\end{tabular}


XI. Organization $E$

A. Summary of Organization Description

1. Staff: 6 programming branches with $19-12$ programmers in each.

2. Hardware: interactive terminals microprocessor minicomputer

3. Programming activities:

All applications programming including editing,weighing, tallying and displaying statistics.

4. Programming languages:

Principal: FORTRAN (98\%)

Other: ALGOL

5. Contractor support: None

6. Programmer recruiting: None reported

7. Quality control:

a. Standards used: Internally developed

b. Guidelines used: None reported

c. Tools used: None reported

d. Comments: No procedure used for quality assurance.

B. Summary of Individual Programmer Profiles

1. Total number surveyed: 57

2. Age summary: No. \&

$\begin{array}{lll}4 \emptyset \text { and over } & 12 & 21 \\ 3 \emptyset-39 & 17 & 3 \emptyset \\ 25-29 & 18 & 32 \\ 2 \varnothing-24 & 1 \varnothing & 17\end{array}$


3. Grade Summary:

No. $\quad$ o

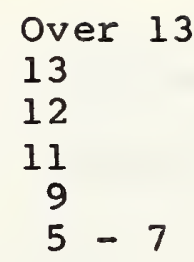

$\begin{array}{rr}5 & 9 \\ 8 & 14 \\ 20 & 36 \\ 10 & 17 \\ 7 & 12 \\ 7 & 12\end{array}$

4. Time-in-agency:

$\begin{array}{lrl}\text { Over } 12 \text { yrs. } & 12 & 21 \\ 8-12 \text { yrs. } & 7 & 12 \\ 3-8 \text { yrs. } & 15 & 26 \\ 1-3 \text { yrs. } & 17 & 3 \emptyset \\ 6-12 \text { mos. } & 6 & 11\end{array}$

5. Education summary:

$\begin{array}{lrl}\text { Master's Degree } & 6 & 11 \\ \text { Bachelor's Degree } & 43 & 75 \\ \text { High School Grad. } & 8 & 14\end{array}$

6. Computer system used: large mainframe minicomputer

7. Reported input method for programming:

Batch: $35 \%$

Interactive: $65 \%$

8. Type of programming activity: (order of area in which most time is reportedly spent)

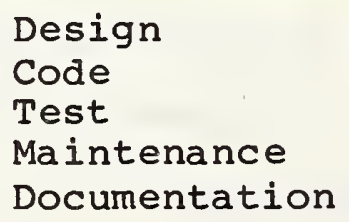

9. Average reported time spent by phase of application programming:

$\begin{array}{lr}\text { New development } & 47 \% \\ \text { Conversion } & 6 \% \\ \text { New versions of old programs } & 23 \% \\ \text { Maintenance } & 12 \% \\ \text { Other } & 12 \%\end{array}$


C. Tabulation of Programmer Profile Data

\begin{tabular}{|c|c|c|c|}
\hline & ge & \multicolumn{2}{|c|}{ Grade } \\
\hline & $\begin{array}{l}\theta+ \\
\theta+ \\
\theta+ \\
\theta+\end{array}$ & $\begin{array}{r}\text { Over } \\
13 \\
13 \\
13\end{array}$ & 13 \\
\hline & $\begin{array}{l}\theta+ \\
\theta+\end{array}$ & $\begin{array}{l}13 \\
12\end{array}$ & \\
\hline & $8+$ & 12 & \\
\hline & $9+$ & 12 & \\
\hline & $\theta+$ & $\begin{array}{l}12 \\
12\end{array}$ & \\
\hline & $\theta+$ & $\begin{array}{l}12 \\
12\end{array}$ & \\
\hline $\begin{array}{l}30 \\
3 \emptyset\end{array}$ & $\begin{array}{l}-39 \\
-\quad 39\end{array}$ & $\begin{array}{l}\text { Over } \\
\text { Over }\end{array}$ & $\begin{array}{l}13 \\
13\end{array}$ \\
\hline $\begin{array}{l}30 \\
30\end{array}$ & $\begin{array}{l}-39 \\
-\quad 39\end{array}$ & $\begin{array}{l}\text { Over } \\
\text { Over }\end{array}$ & $\begin{array}{l}13 \\
13\end{array}$ \\
\hline $\begin{array}{l}30 \\
3 \varnothing\end{array}$ & $\begin{array}{l}-39 \\
-\quad 39\end{array}$ & $\begin{array}{l}13 \\
13\end{array}$ & \\
\hline 30 & -39 & 13 & \\
\hline 30 & -39 & 12 & \\
\hline 30 & $\begin{array}{l}39 \\
-\quad 39\end{array}$ & 12 & \\
\hline $\begin{array}{l}3 \emptyset \\
30\end{array}$ & $\begin{array}{l}-39 \\
-\quad 39\end{array}$ & $\begin{array}{l}12 \\
12\end{array}$ & \\
\hline 30 & -39 & 12 & \\
\hline 30 & -39 & 12 & \\
\hline 30 & -39 & 11 & \\
\hline $\begin{array}{l}30 \\
30\end{array}$ & $\begin{array}{l}-39 \\
-\quad 39\end{array}$ & $\begin{array}{l}9 \\
9\end{array}$ & \\
\hline 30 & -39 & $5-$ & 7 \\
\hline 25 & -29 & 13 & \\
\hline 25 & -29 & 12 & \\
\hline 25 & -29 & 12 & \\
\hline 25 & -29 & 12 & \\
\hline 25 & -29 & 12 & \\
\hline 25 & -29 & 12 & \\
\hline 25 & -29 & 12 & \\
\hline 25 & -29 & 12 & \\
\hline 25 & -29 & 11 & \\
\hline 25 & -29 & 11 & \\
\hline 25 & -29 & 11 & \\
\hline 25 & -29 & 11 & \\
\hline 25 & -29 & 11 & \\
\hline 25 & -29 & 9 & \\
\hline 25 & -29 & 9 & \\
\hline
\end{tabular}

Time-in-agency

Education

8 - 12 yrs. M

8 - 12 yrs. B

Over 12 yrs. HS

8 - 12 yrs.

Over 12 yrs.

B

Over 12 yrs.

Over 12 yrs.

Over 12 yrs.

Over 12 yrs.

3 - 8 yrs.

8 - 12 yrs.

Over 12 yrs.

8 - 12 yrs.

8 - 12 yrs.

Over 12 yrs.

Over 12 yrs.

8 - 12 yrs.

3 - 8 yrs.

1 - 3 yrs.

3 - 8 yrs.

3 - 8 yrs.

over 12 yrs.

3 - 8 yrs.

Over 12 yrs.

Over 12 yrs.

1 - 3 yrs.

3 - 8 yrs.

1 - 3 yrs.

1 - 3 yrs.

3 - 8 yrs.

3 - 8 yrs.

3 - 8 yrs.

3 - 8 yrs.

3 - 8 yrs.

3 - 8 yrs.

3 - 8 yrs.

3 - 8 yrs.

3 - 8 yrs.

1 - 3 yrs.

1 - 3 yrs.

1 - 3 yrs.

1 - 3 yrs.

1 - 3 yrs.

1 - 3 yrs.

B

HS

HS

HS

M

B

B

B

B

B

B

B

M

B

B

HS

B

B

B

HS

$M$

HS

B

B

M

B

B

B

B

B

B

B

B

B

B

B

B

B

B 
C. Tabulation of Programmer Profile Data (continued)

Age
$25-29$
$25-29$
$25-29$
$2 \emptyset-24$
$2 \emptyset-24$
$2 \emptyset-24$
$2 \emptyset-24$
$2 \emptyset-24$
$2 \emptyset-24$
$2 \emptyset-24$
$2 \emptyset-24$
$2 \emptyset-24$
$2 \emptyset-24$

Grade

$5-7$

$5-7$

$5-7$

11

11

11

11

9

9

9

$5-7$

$5-7$

$5-7$
Time-in-agency

6 - 12 mos.

$6-12$ mos.

$6-12$ mos.

1 - 3 yrs.

1 - 3 yrs.

6 - 12 mos.

1 - 3 yrs.

1 - 3 yrs.

1 - 3 yrs.

1 - 3 yrs.

6 - 12 mos.

1 - 3 yrs.

6 - 12 mos.
Education

B
M
B
B
B
B
B
B
B
B
B
B
B 
XII. Organization $F$

A. Summary of Organization Description

1. Staff: Graphics Software Branch

Data Base Management Systems Branch

2. Hardware: mainframes

CRT terminals

graphics terminals

hard copy terminals

3. Programming activities:

They cover the full range of programming for graphics and photocomposition, data base management and generalized software for scientific applications.

4. Programming languages:

\begin{tabular}{llll} 
& Gen. Software & Graphics & Data Base \\
\cline { 2 - 3 } & COBOL & FORTRAN & COBOL \\
Other: & & ALGOL & FORTRAN \\
& & & Assembly
\end{tabular}

5. Contractor support:

Data Base support Graphic procedure supp.

$\$ 150, \varnothing \varnothing \varnothing / \mathrm{yr}$ \& \& 3 man-yrs.

( 58 of programming for data base done by contractors)

6. Programmer recruiting:

Uses regular OPM and agency personnel support and procedures. College recruiting is accomplished with the aid of special programs.

7. Quality control:

a. Standards used: ASCII, FORTRAN, and COBOL; FIPS standards and structured programming techniques are generally used.

b. Guidelines used: Same as a. 
c. Tools used: Interactive editor, language proçedure libraries, and document processor

d. Comments: Use procedures for quality assurance at module level; need tools for better documentation of individual programs.

B. Summary of Individual Programmer Profiles

1. Total number surveyed: 25

2. Age summary: No. 8

$$
\begin{aligned}
& 4 \varnothing \text { and over } \\
& 3 \varnothing=39 \\
& 25=29 \\
& 2 \varnothing-24
\end{aligned}
$$

8

$8 \quad 32$

$8-32$

3. Grade summary:

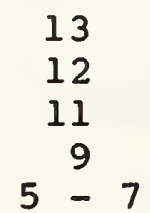

4. Time-in-agency summary:

$$
\begin{aligned}
& \text { Over } 12 \text { yrs. } \\
& 8-12 \text { yrs. } \\
& 3-8 \text { yrs. } \\
& 1-3 \text { yrs. } \\
& 6-12 \text { mos. }
\end{aligned}
$$

5. Education summary:

$\begin{array}{lrl}\text { Master's Degree } & 5 & 2 \emptyset \\ \text { Bachelor's Degree } & 15 & 69 \\ \text { High School Grad. } & 5 & 2 \emptyset\end{array}$

6. Computer system used: Large mainframe Minicomputer

7. Reported input method for programming: 
8. Type of programming activity: (order of area in which most time is reportedly spent)

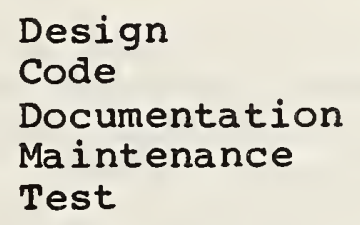

9. Average reported time spent by phase of application programming:

$\begin{array}{lr}\text { New development } & 52 \% \\ \text { Conversion } & 5 \% \\ \text { New versions of old programs } & 17 \% \\ \text { Maintenance } & 26 \% \\ \text { Other } & \emptyset\end{array}$

C. Tabulation of Programmer Profile Data

\begin{tabular}{|c|c|c|c|c|}
\hline & Age & Grade & Time-in-agency & Education \\
\hline & $\begin{array}{l}40+ \\
4 \emptyset+ \\
4 \varnothing+ \\
4 \varnothing+\end{array}$ & $\begin{array}{l}13 \\
13 \\
13 \\
13\end{array}$ & $\begin{array}{l}1-3 \text { yrs. } \\
1-3 \text { yrs } \\
1-3 \text { yrs } \\
8-12 \text { yrs. }\end{array}$ & $\begin{array}{l}B \\
B \\
B \\
M\end{array}$ \\
\hline & $\begin{array}{l}40+ \\
49+ \\
49+\end{array}$ & $\begin{array}{r}13 \\
9 \\
9\end{array}$ & $\begin{array}{l}3 \text { - } 8 \text { yrs. } \\
\text { Over } 12 \text { yrs. } \\
6 \text { - } 12 \text { mos. }\end{array}$ & $\begin{array}{r}B \\
\mathrm{HS} \\
B\end{array}$ \\
\hline & $40+$ & 9 & $1-3$ yrs. & HS \\
\hline $\begin{array}{l}30 \\
30\end{array}$ & $\begin{array}{l}-39 \\
-\quad 39\end{array}$ & $\begin{array}{l}12 \\
12\end{array}$ & $\begin{array}{l}3-8 \text { yrs. } \\
3-8 \text { yrs. }\end{array}$ & $\begin{array}{l}\mathrm{B} \\
\mathrm{M}\end{array}$ \\
\hline $\begin{array}{l}30 \\
30\end{array}$ & $\begin{array}{l}-\quad 39 \\
-\quad 39\end{array}$ & $\begin{array}{l}12 \\
12\end{array}$ & $\begin{array}{l}3-8 \text { yrs. } \\
1-3 \text { yrs. }\end{array}$ & $\begin{array}{r}\text { B } \\
\text { HS }\end{array}$ \\
\hline 30 & -39 & 12 & $1-3$ yrs. & B \\
\hline 30 & -39 & 11 & $6-12$ mos. & HS \\
\hline 30 & -39 & 9 & $1-3$ yrs. & M \\
\hline $3 \emptyset$ & -39 & $5-7$ & $6-12$ mos. & HS \\
\hline 25 & -29 & 13 & 1 - 3 yrs. & B \\
\hline 25 & -29 & 12 & 3 - 8 yrs. & B \\
\hline 25 & -29 & 12 & $6-12$ mos. & B \\
\hline 25 & -29 & 12 & 1 - 3 yrs. & $M$ \\
\hline 25 & -29 & 11 & 3 - 8 yrs. & B \\
\hline 25 & -29 & 11 & $6-12$ mos. & B \\
\hline 25 & -29 & 9 & $6-12$ mos. & $\bar{M}$ \\
\hline 25 & -29 & 9 & $1-3$ yrs. & B \\
\hline $2 \varnothing$ & -24 & 11 & 3 - 8 yrs. & B \\
\hline
\end{tabular}


XII. Organization $G$

A. Summary of Organization Description

1. Staff: Operating Systems Branch

Peripheral Systems Branch

Programming Assistance and 10

Languages Branch

User Training and Info. Branch 14

Management and staff support 11

2. Hardware: large mainframes

small mainframe

3. Programming activities:

Operating system (OS) and system support software

4. Programming languages:

Principa 1: Assembly

Other: FORTRAN and COBOL

5. Contractor support:

About $2 \%$ of programming done by contractor.

6. Programmer recruiting:

Only comment given that universities are primary sources of new employees.

7. Quality control:
a. Standards used: FIPS
b. Guidelines used: FIPS when possible
c. Tools used: No comment
d. Comments: There is a comprehensive procedure for quality assurance.

B. Summary of Individual Programmer Profiles

1. Total number surveyed: 8

2. Age summary:

No. $\quad \%$

$$
\begin{aligned}
& 4 \varnothing \text { and over } \\
& 3 \varnothing-39 \\
& 25-29 \\
& 2 \varnothing-24
\end{aligned}
$$


3. Grade summary:

$$
\begin{array}{r}
12 \\
11 \\
5-7
\end{array}
$$

No.

8

$\begin{array}{ll}3 & 37 \\ 3 & 37 \\ 2 & 26\end{array}$

4. Time-in-agency summary:

$\begin{array}{lll}\text { Over } 12 \text { yrs. } & 1 & 13 \\ 8-12 \text { yrs. } & 3 & 37 \\ 3-8 \text { yrs. } & 2 & 25 \\ 1-3 \text { yrs. } & 2 & 25 \\ 6-12 \text { mos. } & \end{array}$

5. Education summary:

Master's Degree

Bachelor's Degree

High School Grad.

6. Computer system used: large mainframe small mainframe

7. Reported input method for programming:

Batch: $22 \%$

Interactive: 788

8. Type of programming activity: (order of area in which most time is reportedly spent)

Code

Maintenance

Test

Design

Documentation

9. Average reported time spent by phase of system programming:

New development 
C. Tabulation of Programmer Profile Data

$\begin{array}{cc}\text { Age } & \text { Grade } \\ 4 \emptyset+ & 5-7 \\ 3 \emptyset-39 & 12 \\ 3 \emptyset-39 & 12 \\ 3 \emptyset-39 & 11 \\ 25-29 & 12 \\ 25-29 & 11 \\ 25-29 & 11 \\ 2 \varnothing-24 & 5-7\end{array}$

Time-in-agency

1 - 3 yrs.

Over 12 yrs.

3 - 8 yrs.

6 - 12 mos.

3 - 8 yrs.

1 - 3 yrs.

3 - 8 yrs.

6 - 12 mos.
Education

$\mathrm{B}$

HS

B

M

HS

B

HS

B 
XIV. Organization $\mathrm{H}$

A. Summary of Organization Description

1. Staff: Administration 3

Programming Branch $1 \quad 18$

Programing Branch $2 \quad 10$

Programming Branch $3 \quad 15$

2. Hardware: large mainframes remote terminals printers word processors

3. Programming activities:

Entire staff involved in information processing associated with statistical surveys. Programming defined as applications support.

4. Programming languages:

Principal: FORTRAN

Other: ALGOL

5. Contractor support: None

6. Programmer recruiting:

Emphasis is on college recruitment for graduates in computer science or math. Low pay scale and competition from the private sector have combined to make such recruiting difficult.

7. Quality control:
a. Standards used: ASCII FORTRAN b. Guidelines used: Internal controls for mnemonics,
specifications, documentation, etc.,
c. Tools used: Various software developed by vendors and in-house staff.
d. Comments: Comprehensive procedures ae used for quality assurance. Easy-to-use on-lin
debugging/analysis routines are needed. 
B. Summary of Individual Programmer Profiles

1. Total number surveyed: 31

2. Age summary: No.

$4 \emptyset$ and over
$3 \emptyset-39$
$25-29$
$2 \varnothing-24$

$\begin{array}{rr}5 & 16 \\ 9 & 29 \\ 13 & 42 \\ 4 & 13\end{array}$

3. Grade Summary:

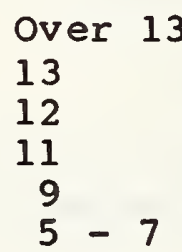

$\begin{array}{ll}3 & 1 \varnothing \\ 6 & 19 \\ 4 & 13 \\ 5 & 16 \\ 8 & 26 \\ 5 & 16\end{array}$

4. Time-in-agency summary:

$$
\begin{aligned}
& \text { Over } 12 \text { yrs. } \\
& 8-12 \text { yrs. } \\
& 3-8 \text { yrs. } \\
& 1-3 \text { yrs. } \\
& 6-12 \text { mos. }
\end{aligned}
$$

$\begin{array}{rr}5 & 16 \\ 2 & 6 \\ 8 & 26 \\ 14 & 46 \\ 2 & 6\end{array}$

5. Education summary:

$\begin{array}{lrr}\text { PhD } & 1 & 3 \\ \text { Master's Degree } & 5 & 16 \\ \text { Bachelor's Degree } & 22 & 71 \\ \text { High School Grad. } & 3 & 1 \emptyset\end{array}$

6. Computer system used: large mainframe

7. Reported input method for programming:

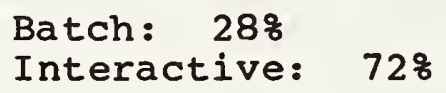

8. Type of programming activity: (order of area in which most time is reportedly spent)

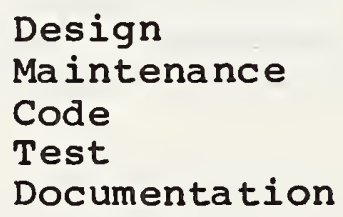


9. Average reported time spent by phase of application programming:

$\begin{array}{lc}\text { New development } & 47 \% \\ \text { Conversion } & 10 \% \\ \text { New versions of old programs } & 19 \% \\ \text { Maintenance } & 24 \% \\ \text { Other } & \emptyset\end{array}$

C. Tabulation of Programmer Profile Data

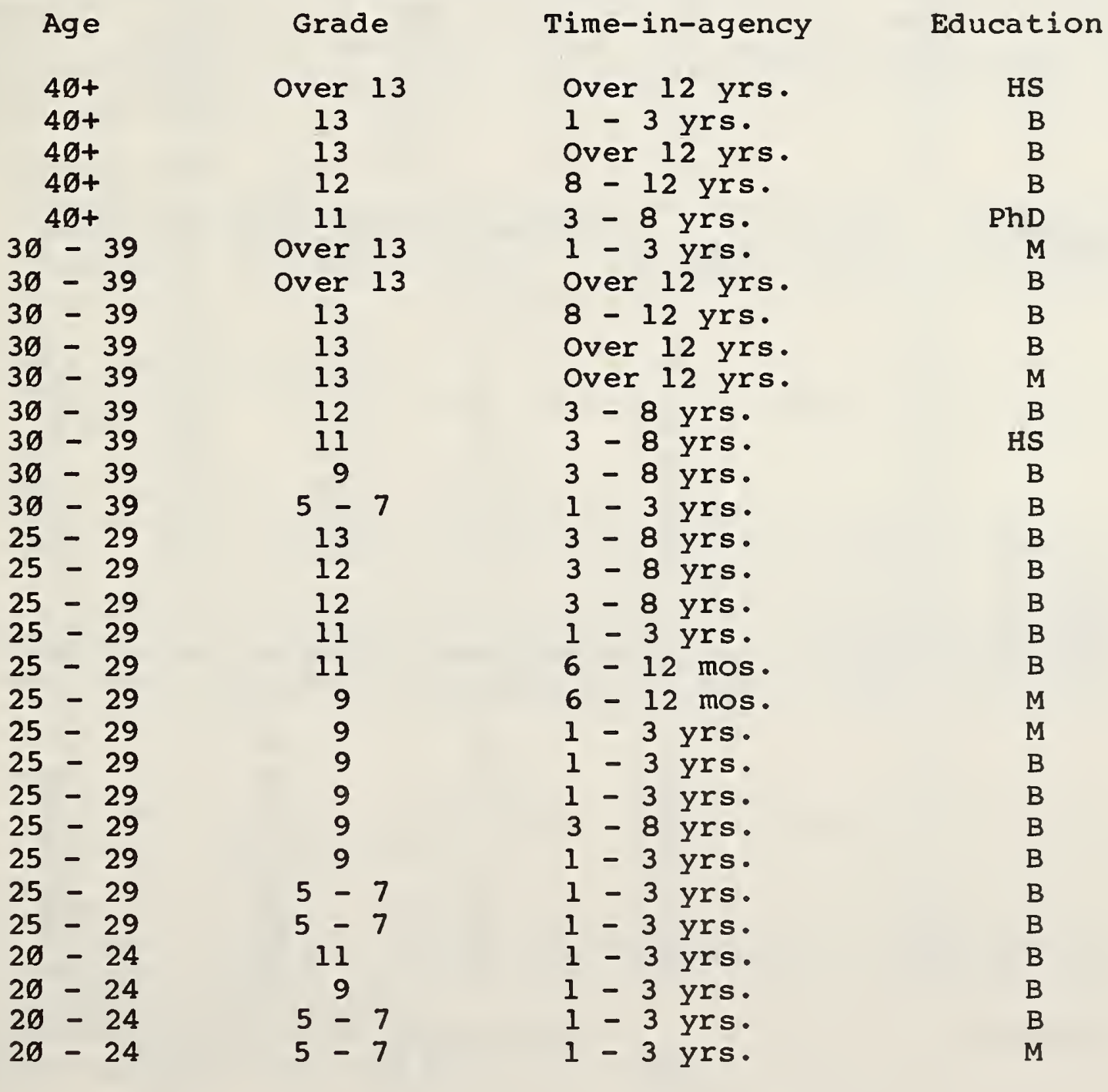


Chart 1

Tabulations of Percentages from Computer Programmer Profile Statistics.

Organization

A. Ages

$$
40 \& \text { over } \begin{gathered}
\text { Percentages by Age Groupings } \\
3 \emptyset-39
\end{gathered} \quad \begin{aligned}
& 25-29 \\
& 2 \emptyset-24
\end{aligned}
$$

$\begin{array}{ll}\text { A } & 40 \\ \text { B } & 14 \\ \text { C } & 43 \\ \text { D } & 27 \\ \text { E } & 21 \\ \text { F } & 32 \\ \text { G } & 13 \\ \text { H } & 16\end{array}$

52
42
45
17
30
32
37
29

4

36

12

39

32

32

37

42

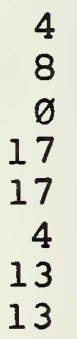

13

B. Grades

\begin{tabular}{ccccrr} 
Over 13 & $\begin{array}{c}\text { Percentages } \\
\text { by Grade }\end{array}$ & \multicolumn{4}{l}{ Groupings } \\
& 13 & 12 & 11 & 9 & $5-7$ \\
$\emptyset$ & 43 & 22 & 22 & 4 & 8 \\
9 & 8 & 42 & 14 & 6 & $3 \emptyset$ \\
$\emptyset$ & 19 & 45 & 6 & 21 & $\emptyset$ \\
9 & 22 & 27 & 17 & 17 & 17 \\
$\emptyset$ & 14 & 36 & 17 & 12 & 12 \\
$\emptyset$ & 24 & 32 & 16 & 24 & 4 \\
$1 \emptyset$ & $\emptyset$ & 37 & 37 & $\emptyset$ & 26 \\
& 19 & 13 & 16 & 26 & 16
\end{tabular}

C. Time-in-agency

Percentages by Time-in-agency Groupings $>12$ yrs. 8-12 yrs. 3-8 yrs. 1-3 yrs. <1 yr.

$\begin{array}{rr}\text { A } & 4 \\ \text { B } & 8 \\ \text { C } & 18 \\ \text { D } & 22 \\ \text { E } & 21 \\ \text { F } & 4 \\ \text { G } & 13 \\ \text { H } & 16\end{array}$

22
17
24
6
12
4
$\varnothing$
6

$4 \emptyset$
39
43
22
26
28
37
26

26

33

9

$5 \varnothing$

$3 \emptyset$

$4 \varnothing$

25

46

8
3
6
$\varnothing$
11
24
25
6

Percentages by Levels of Education M.A.

B.A.

High School
$\mathrm{PhD}$
A

B

C

D

E

F

G

$\mathrm{H}$ 


\title{
STATISTICS FROM THE GENERAL PERSONNEL DATA FILE OF THE
}

\author{
OFFICE OF PERSONNEL MANAGEMENT
}

1. The Office of Personnel Management (OPM) was requested to provide personnel statistics pertinent to this study of computer programmers from their september 1980 data base. The Work Force Information Division of OPM assisted in the preparation of the detailed request that was submitted for computer retrieval from the Central Personnel Data File. The following criteria were used for data selection:

a. Occupational code:

Data only from records that have occupational code of 334 (Computer specialist) which includes computer programmers, computer systems analysts, computer equipment analysts, and combinations of these skills.

b. Work schedule code:

Data only from records where this code is full-time employees

c. Standard Metropolitan Statistical Area:

Data only from records where the code for this element is for the washington Metropolitan area which includes the District of Columbia; Arlington, Fairfax, Loudoun and Prince William counties, and Alexandria, Fairfax, Manassas, Manassas Park and Falls Church cities in Virginia; Charles, Montgomery and Prince Georges counties in Maryland.

d. Agency code:

Exclude records where this code represents one of the military departments

The criteria were later expanded to include the occupational code of 330 (Digital Computer Systems Administration), although this data were kept separate in the statistical summaries and have not been analyzed for this study.

2. The data requested from the qualifying records were as follows:
a. Agency and Sub-agency
b. Date of birth
c. GS equivalent
d. Salary
e. Sex
f. Supervisory or non-supervisory
g. Academic education level 
3. The OPM was also requested to prepare three statistical summaries for each Sub-agency code, using the same criteria as previously listed.

These were:

a. Age: (numbers by groupings)

$$
\begin{aligned}
& \text { Under } 2 \emptyset \\
& 2 \emptyset-24 \\
& 25-29 \\
& 3 \emptyset-39 \\
& 4 \varnothing \text { and over }
\end{aligned}
$$

b. GS equivalents: (numbers by groupings)

$$
\begin{aligned}
& 5-7 \\
& 9 \\
& 11 \\
& 12 \\
& 13 \text { and over }
\end{aligned}
$$

c. Highest academic level: (numbers by groupings)

High school

Bachelor's degree

Master's degree

Doctorate

The tables submitted as attachments to this enclosure present the highlights from these statistical summaries. It is emphasized that these statistics are for the Washington Metropolitan area only, as defined by the standard Metropolitan Statistical Area. Both military and civilian members of the military departments are excluded. The data, as obtained from OPM, are as of September 30, 1980 .

4. The following observations concerning the computer specialists are offered as a result of reviewing the statistics in each table:

a. Over $80 \%$ are $3 \emptyset$ years of age or older;

b. Almost one-half are at the grade level of GS-13 or over;

c. Almost one-half have only a high school education and about two-fifths have a bachelor's degree;

d. The Treasury Department (with the largest number in its employ) has a younger staff than other agencies, with about one-fourth from 25-29 and only about one-fourth 40 or over. The average for these age groups among all agencies, as shown on Table 1 , are 148 and 38\%, respectively. Conversely, the age level in the Department of Housing and Urban Development is usually high, i.e. $6 \%$ and $47 \%$ for these two age groupings. 
e. The Treasury Department has a higher percentage of lower grades than the average of all agencies. Almost threefourths of the computer specialists in the Department of Energy and Housing and Urban Development are GS-13's or higher.

f. The levels of education are remarkably similar among the twelve agencies employing over 28 of the total. However, the General Services Administration has an unusually high percentage with only a high school education.

g. Almost one-half of the total number of computer specialists are employed by the three departments of Treasury, Commerce and Health and Human Services. The organizations with unusually high numbers in these departments are as follows:

HHS :

National Institutes of Health

777

Commerce: Nat. Oceanic \& Amos. Admin.

291

234

List of Tables:

TABLE 1. Number and Percentage of Computer Specialists (Series 334) by Age, Grade and Education.

TABLE 2. Number and Percentage of Computer Specialists by Age in These Agencies Employing Over 28 of Total Number.

TABLE 3. Number and Percentage of Computer Specialists by Grade in Those Agencies Employing Over $2 \%$ of Total Number.

TABLE 4. Number and Percentage of Computer Specialists by Education in Those Agencies Employing Over 28 of Total Number.

TABLE 5. Number and percent of Computer Specialists by Agency.

TABLE 6. Number of Computer Specialists by Age Groupings for Agencies and Sub-agencies.

TABLE 7. Number of Computer Specialists by Grade Levels for Agencies and Sub-agencies.

TABLE 8. Number of Computer Specialists by Education Levels for Agencies and Sub-agencies. 
Appendix 1

The following organizational abbreviations are used in Tables 1-8

\begin{tabular}{|c|c|}
\hline AID : & Agency for International Development \\
\hline BEA: & Bureau of Economic Analysis \\
\hline CAB : & Civil Aeronautics Board \\
\hline CPSC: & Consumer Product Safety Commission \\
\hline CSA: & Community Services Administration \\
\hline DoC : & Commerce, Department of \\
\hline DOE : & Energy, Department of \\
\hline DoI : & Interior, Department of \\
\hline DOL: & Labor, Department of \\
\hline DOT : & Transportation, Department of \\
\hline EDA: & Economic Development Administration \\
\hline EPA : & Environmental Protection Agency \\
\hline FAA : & Federal Aviation Administration \\
\hline FBI: & Federal Bureau of Investigation \\
\hline FCC : & Federal Communications Commission \\
\hline FDA: & Food and Drug Administration \\
\hline FDIC: & Federal Deposit Insurance Corporation \\
\hline FHA: & Federal Highway Administration \\
\hline FHLB : & Federal Home Loan Bank Board \\
\hline FTC: & Federal Trade Commission \\
\hline GSA: & General Services Administration \\
\hline HHS : & Health and Human Services, Department of \\
\hline HUD : & Housing and Urban Development, Department of \\
\hline ICA: & International Communication Agency \\
\hline ICC: & Interstate Commerce Commission \\
\hline IDCA: & International Development Cooperation Agency, U.S. \\
\hline IRS : & Internal Revenue Service \\
\hline MBD : & Minority Business Development Agency \\
\hline NASA: & National Aeronautics and Space Administration \\
\hline NBS : & National Bureau of Standards \\
\hline $\mathrm{NIH}:$ & National Institutes of Health \\
\hline NLRB : & National Labor Relations Board \\
\hline NOAA: & National Oceanic and Atmospheric Administration \\
\hline NRC: & Nuclear Regulatory Commission \\
\hline NSF : & National Science Foundation \\
\hline NTIS : & National Technical Information Service \\
\hline OPM : & Office of Personnel Management \\
\hline SBA: & Small Business Administration \\
\hline SEA : & Science and Education Administration \\
\hline SEC : & Securities and Exchange Commission \\
\hline SSA: & Social Security Administration \\
\hline USDA: & Agriculture, United States Department of \\
\hline VA: & Veterans Administration \\
\hline
\end{tabular}

Tables 5-8 are alphabetical by agency according to the standards used in automated interchange (FPM Supplement 292-1), e.g • USDA is alphabetized by Agriculture, IDCA is alphabetized by United States International Development Cooperation Agency. 
TABLE 1. Number and Percentage of Computer Specialists (Series 334) by Age, Grade and Education.

\begin{tabular}{|c|c|c|c|c|c|c|c|c|c|}
\hline Age & 20 & or & less & $21-24$ & $25-29$ & $30-39$ & $4 \varnothing$ & and over & Total \\
\hline Number & & 5 & & 184 & 869 & 2678 & & 2273 & 600 \\
\hline Percent & & - & & 3 & 14 & 45 & & 38 & 10 \\
\hline
\end{tabular}

$\begin{array}{lccccrrrr}\text { Grade } & 5 & 7 & 9 & 11 & 12 & 13 \text { and over } & \text { Total } \\ \text { Number } & 23 \emptyset & 44 \emptyset & 473 & 591 & 1494 & 2718 & 5946 \\ \text { Percent } & 4 & 7 & 8 & 1 \emptyset & 25 & 46 & 1 \emptyset \emptyset\end{array}$

$\begin{array}{lcccrr}\text { Education } & \text { High School } & \text { B.A. } & \text { M.A. } & \text { PhD. } & \text { Total } \\ \text { Number } & 2832 & 2435 & 541 & 31 & 5839 \\ \text { Percent } & 48 & 42 & 9 & 1 & 1 \emptyset \emptyset\end{array}$

Note: The totals in the three areas differ. These are the figures as submitted by OPM and no attempt has been made to reconcile the data. The differences are not great enough, however, to affect the analysis. 
TABLE 2. Number and Percentage of Computer Specialists by Age in Those Agencies Employing Over $2 \%$ of Total Number.

Agency

Age

$2 \varnothing$ or less 21-24 25-29 $30-39 \quad 4 \varnothing \&$ over Total

Treasury

Number

Percent

DoC

Number

Percent

HHS

Number

Percent

USDA

Number

Percent

DOL

Number

Percent

Justice

Number

Percent

VA

Number

Percent

GSA

Number

Percent

1

$-$

45

4

277
24

528

47

285

25

372

40

17

355

38

65

9

320

44

44

11

198

52

134

35

329

45

728

100

$\varnothing$

2

10

3

52

16

141

44

118

37

321

100

38

12

154

50

107

34

311

100

31

13

111

45

99

$4 \varnothing$

247

100

27

11

113

99

244

2

46

41

100

DOT

Number

Percent

35

DOE

Number

Percent

5
2

98

43

92

$4 \varnothing$

230

$\emptyset$

15

14

99

49

88

44

201

7

$10 \varnothing$

HUD

Number

Percent

12

86

47

88

47

DoI

Number

Percent 
TABLE 3. Number and Percentage of Computer Specialists by Grade in Those Agencies Employing Over 28 of Total Number.

Agencies Grades

$\begin{array}{llllllll}5 & 7 & 9 & 11 & 12 & 13 & \text { \& over } & \text { Total }\end{array}$

Treasury

Number

Percent

$\begin{array}{lllll}113 & 151 & 101 & 84 & 276\end{array}$

419

37

1144

$\begin{array}{lllll}1 \varnothing & 13 & 9 & 7 & 24\end{array}$

$10 \varnothing$

DoC

Number

Percent

$\begin{array}{rr}21 & 63 \\ 2 & 7\end{array}$

$82 \quad 116 \quad 252$

410

43

944

$1 \varnothing 0$

HHS

Number

Percent

$\begin{array}{rr}16 & 33 \\ 2 & 5\end{array}$

$\begin{array}{lll}63 & 82 & 199\end{array}$

330

45

723

$\begin{array}{lll}9 & 11 & 28\end{array}$

$1 \varnothing \emptyset$

USDA

Number

Percent

$\begin{array}{rrrrr}5 & 23 & 3 \emptyset & 34 & 85 \\ 1 & 6 & 8 & 1 \emptyset & 24\end{array}$

181

51

358

100

DOL

Number

Percent

$1134 \quad 3$

$31 \quad 48 \quad 96$

$1 \varnothing 5$

325

$\begin{array}{lllll}3 & 10 & 10 & 15 & 3 \emptyset\end{array}$

$1 \varnothing \emptyset$

Justice

Number

Percent

$\begin{array}{rr}7 & 23 \\ 2 & 7\end{array}$

$21 \quad 26 \quad 63$

172

56

312

820

$1 \varnothing \varnothing$

VA

Number

Percent

$\begin{array}{rrrrr}9 & 21 & 16 & 11 & 53 \\ 4 & 8 & 6 & 4 & 21\end{array}$

139

57

249

$1 \varnothing \varnothing$

GSA

Number

Percent

$3 \quad 16 \quad 21$

$27 \quad 101$

81

33

249

$8 \quad 11 \quad 41$

$1 \varnothing \varnothing$

DOT

Number

Percent

$16 \quad 9 \quad 28$

$21 \quad 42$

113

$5 \varnothing$

229

$10 \varnothing$

DOE

Number

Percent

$\emptyset$

3
2

$\begin{array}{lll}5 & 19 & 26\end{array}$

144

73

197

$10 \quad 13$

100

HUD

Number

13

2

$12 \quad 29$

140

187

Percent

- 21

616

100

DoI

Number

$\emptyset$

6

7 
TABLE 4. Number and Percentage of Computer specialists by Education in Those Agencies Employing Over $2 \%$ of Total Number.

Agency

Education
High School
B.A.
M.A.
PhD.
Total

Treasury

Number

Percent

DoC

Number

Percent

389

42

435

47

91

10

9

1

924

100

HHS

Number

Percent

343

49

283

$4 \varnothing$

76

11

$7 \varnothing 4$

100

USDA

Number

Percent

166

46

143

40

46

13

358

100

DoL

Number

Percent

156

50

124

$4 \varnothing$

27

9

309

$10 \varnothing$

Justice

Number

Percent

156

119

51

32

10

310

100

VA

Number

Percent

121

50

102

42

19

8

243

$1 \varnothing \varnothing$

GSA

Number

Percent

150

61

78

32

18

7

$\varnothing$

246

$10 \varnothing$

DOT

Number

116

51

93

Percent

$4 \varnothing$

20

9

$\emptyset$

229

100

DOE

Number

Percent

HUD

Number

80

$\emptyset$

$\emptyset$

DoI

Number

64

73

20

13

2

159

46 
TABLE 5. Number and Percent of Computer Specialists by Agency.

\begin{tabular}{|c|c|c|c|c|c|}
\hline Agency & No. & $\begin{array}{l}8 \text { of } \\
\text { Tota } 1\end{array}$ & Agency & No. & $\begin{array}{l}\& \text { of } \\
\text { Total }\end{array}$ \\
\hline$A C T I O N$ & 27 & $\emptyset .5$ & $I C C$ & 28 & 0.5 \\
\hline USDA & 383 & 6.4 & Justice & 311 & 5.2 \\
\hline$C A B$ & 24 & 0.4 & DoL & 321 & 5.3 \\
\hline$D o C$ & 939 & $15 \cdot 5$ & NASA & 71 & 1.2 \\
\hline$C S A$ & 30 & 0.5 & $\begin{array}{l}\text { Nat. Credit } \\
\text { Union Admin. }\end{array}$ & 13 & $\emptyset .2$ \\
\hline CPSC & 16 & 0.3 & $N L R B$ & 16 & 0.3 \\
\hline$E P A$ & 84 & 1.4 & NSF & 34 & 0.6 \\
\hline$D O E$ & $2 \varnothing 1$ & $3 \cdot 3$ & $N R C$ & $3 \varnothing$ & 0.5 \\
\hline FCC & 59 & $1 . \varnothing$ & $O P M$ & 112 & 1.9 \\
\hline$F D / C$ & 59 & 1.0 & $\begin{array}{l}\text { Pension. Benefit } \\
\text { Guaranty Corp. }\end{array}$ & 31 & $\emptyset .5$ \\
\hline $\begin{array}{l}\text { Federal Energy } \\
\text { Management Agency }\end{array}$ & 24 & 0.4 & $S E C$ & 28 & 0.5 \\
\hline FHLB & 32 & 0.5 & $S B A$ & 44 & $\emptyset .7$ \\
\hline FTC & 22 & 0.4 & $\begin{array}{l}\text { Smithsonian } \\
\text { Institution }\end{array}$ & 19 & 0.3 \\
\hline GSA & 244 & $4 \cdot 1$ & State & 38 & 0.6 \\
\hline HHS & 728 & 12.1 & DOT & 230 & 3.8 \\
\hline HUD & 187 & 3.1 & Treasury & 1136 & 18.9 \\
\hline Dol & 163 & 2.7 & $I D C A$ & 54 & 0.9 \\
\hline$I C A$ & 23 & 0.4 & $V A$ & 247 & 4.1 \\
\hline & & & TOTAL & 6009 & 100.0 \\
\hline
\end{tabular}


TABLE 6. Number of Computer Specialists by Age Groupings for Agencies and Sub-agencies.

Age Summary

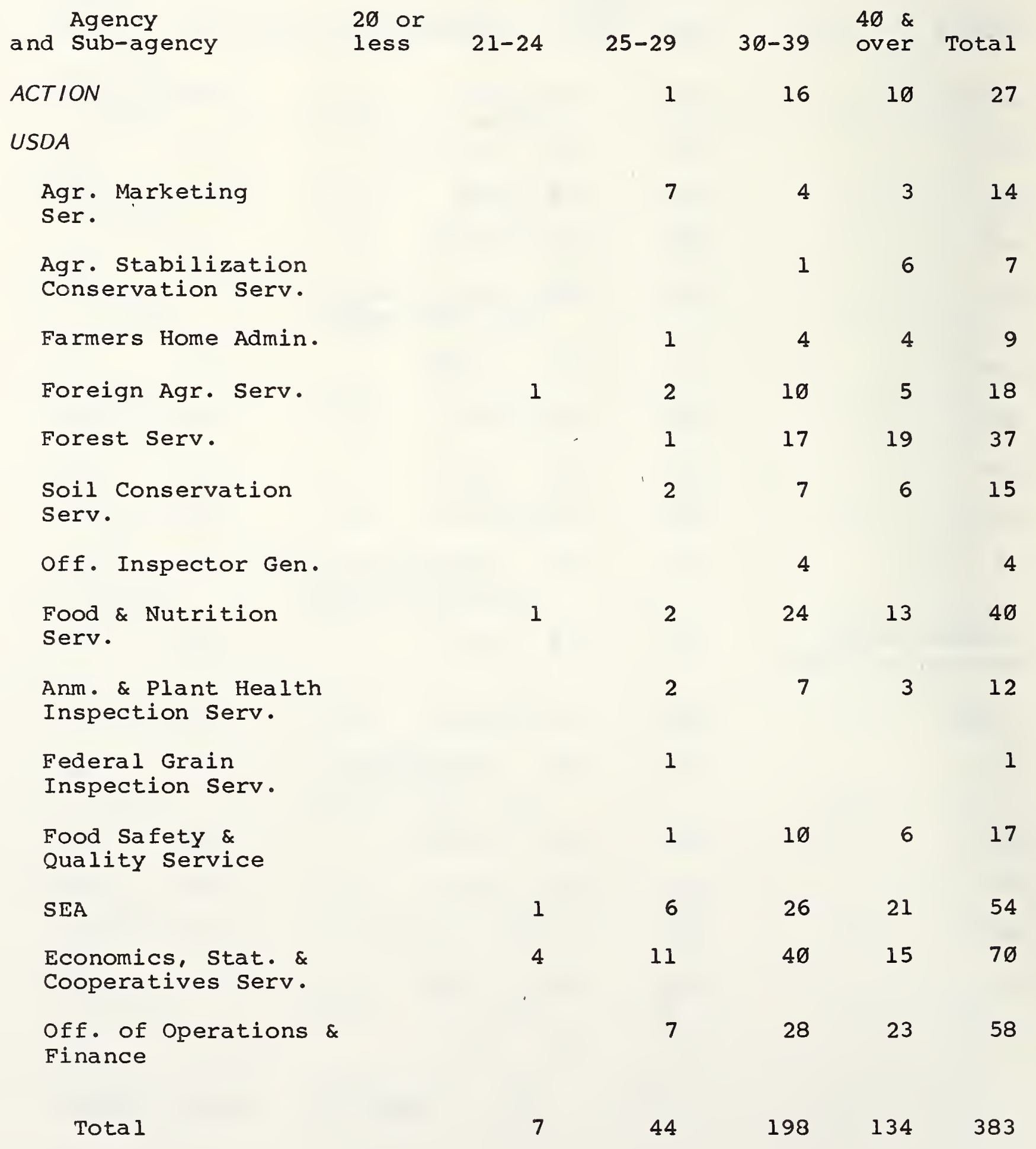


TABLE 6. (continued)

Age Summary

\begin{tabular}{|c|c|c|c|c|c|c|}
\hline $\begin{array}{ll} & \text { Agency } \\
\text { and Sub-agency }\end{array}$ & $\begin{array}{l}20 \text { or } \\
\text { less }\end{array}$ & $21-24$ & $25-29$ & $30-39$ & $\begin{array}{l}4 \emptyset \& \\
\text { over }\end{array}$ & Total \\
\hline$C A B$ & & & & 15 & 9 & 24 \\
\hline \multicolumn{7}{|l|}{ DoC } \\
\hline Off. of secty. & & & 2 & 16 & 23 & 41 \\
\hline EDA & & & 4 & 6 & 11 & 21 \\
\hline BEA & & 2 & 8 & 9 & 3 & 22 \\
\hline NOAA & & 4 & 33 & 92 & 105 & 234 \\
\hline $\begin{array}{l}\text { Internat. \& Trade } \\
\text { Admin. }\end{array}$ & & & 1 & 2 & 3 & 6 \\
\hline Maritime Admin. & & 1 & 5 & 9 & 22 & 37 \\
\hline Patent off. & & 2 & 5 & $2 \varnothing$ & $2 \varnothing$ & 47 \\
\hline NBS & & 4 & 4 & 24 & 43 & 75 \\
\hline MBD & & & & 1 & 2 & 3 \\
\hline $\begin{array}{l}\text { Nat. Telecom. \& } \\
\text { Infor. Agency }\end{array}$ & & & 1 & 4 & 2 & 7 \\
\hline NTIS & & 1 & 2 & 9 & 7 & 19 \\
\hline Bureau of Census & & 38 & 95 & 163 & 130 & 426 \\
\hline $\begin{array}{l}\text { Off. of Federal Stat. } \\
\text { Policy \& Standards }\end{array}$ & & & & & 1 & 1 \\
\hline Total & & 52 & 160 & 355 & 372 & 939 \\
\hline $\operatorname{CSA}$ & & & & 6 & 24 & 30 \\
\hline CPSC & & & & 10 & 6 & 16 \\
\hline$E P A$ & & 6 & 12 & 35 & 31 & 84 \\
\hline$D O E$ & & & 14 & 99 & 88 & 201 \\
\hline$F C C$ & & 4 & 12 & 29 & 14 & 59 \\
\hline FDIC & 1 & 5 & 7 & 25 & 21 & 59 \\
\hline
\end{tabular}


TABLE 6. (continued)

Age Summary

\begin{tabular}{|c|c|c|c|c|c|}
\hline $\begin{array}{cl}\text { Agency } \\
\text { and } \\
\text { Sub-agency }\end{array}$ & $\begin{array}{l}2 \emptyset \text { or } \\
\text { less }\end{array}$ & $21-24$ & $25-29$ & $30-39$ & $\begin{array}{l}4 \varnothing \& \\
\text { over }\end{array}$ \\
\hline $\begin{array}{l}\text { Federal Energy } \\
\text { Management Agency }\end{array}$ & & & & 5 & 19 \\
\hline FHLB & & 1 & 6 & 16 & 9 \\
\hline FTC & & 1 & 4 & 13 & 4 \\
\hline
\end{tabular}

GSA

Off. of Preparedness

Public Bldg. Serv.

4

1

34

Fed. Supply Serv.

15

$10 \quad 29$

Fed. supply serv.

National Archives \&

Records Serv.

1

22

Automated Data \&

5

25

$25 \quad 55$

Telecomm. Serv.

Transport. \& Public

2

24

Utilities Serv.

Off. of Admin.

1

4

17

68

$56 \quad 146$

Off. Of Inspector

General

Total

1

4

27

113

$99 \quad 244$

HHS

Off. of Secty.

3

52

$61 \quad 116$

Off. of Asst.

1

1

14

25

43

Secty. for Health

2

13

15

32

$\&$ Mental Health

1

6

$8 \quad 15$

Health Serv.

Administration 
TABLE 6. (continued)

Age summary

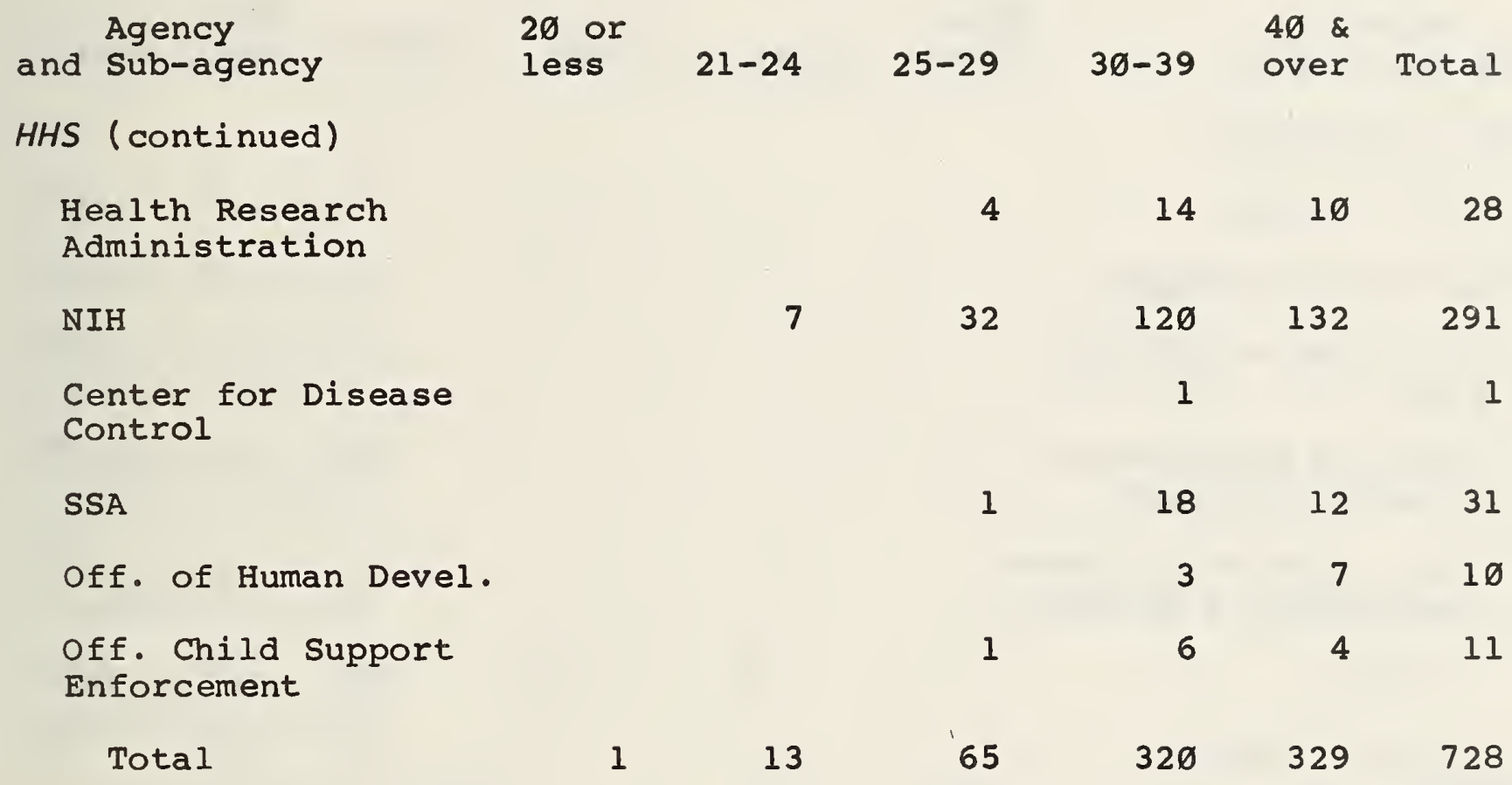

HUD

Asst. Secty. for

1

10

80

81

172

Administration

Asst. Secty for

1

1

1

3

Comm. Plan. Devel.

Fair Housing \&

Equal Opportunity

Asst. Secty for

Housing

other

1

1

12

Total

2

1

86

88

Dol

Off. of Secty.

Bur. of Land Mgmt.

Bur. of Indian Affairs

1

Water \& Power Resources 
TABLE 6. (continued)

Age Summary

$\begin{array}{llllll}\text { Agency } & 2 \varnothing \text { or } & & 40 \& \text { } & \\ \text { and Sub-agency } & \text { less } & 21-24 & 25-29 & 30-39 & \text { over Total }\end{array}$

Dol (continued)

Bur. of Mines

1

8

6

15

National Park Serv.

3

5

4

12

U.S. Fish \& Wildlife

2

12

14

Serv.

Heritage Conservation

4

2

6

\& Recreation Serv.

Off. of Surface Mining

Reclamation \& Enforce.

Total

4

12

74

73

163

Internat. Commun.

8

12

23

Agency

ICC

1

16

11

28

Justice

Offices, Div. \&

1

11

60

34

106

Boards

FBI

1

6

15

33

27

82

Bur. of Prisons

3

17

10

36

Immigration \&

3

2

21

10

36

Naturalization Serv.

Bur. of Prisons

Industries

Drug Enforcement

Administration

Off. of Justice

Assistance

U.S. Marshals Serv. 
TABLE 6. (continued)

Age Summary

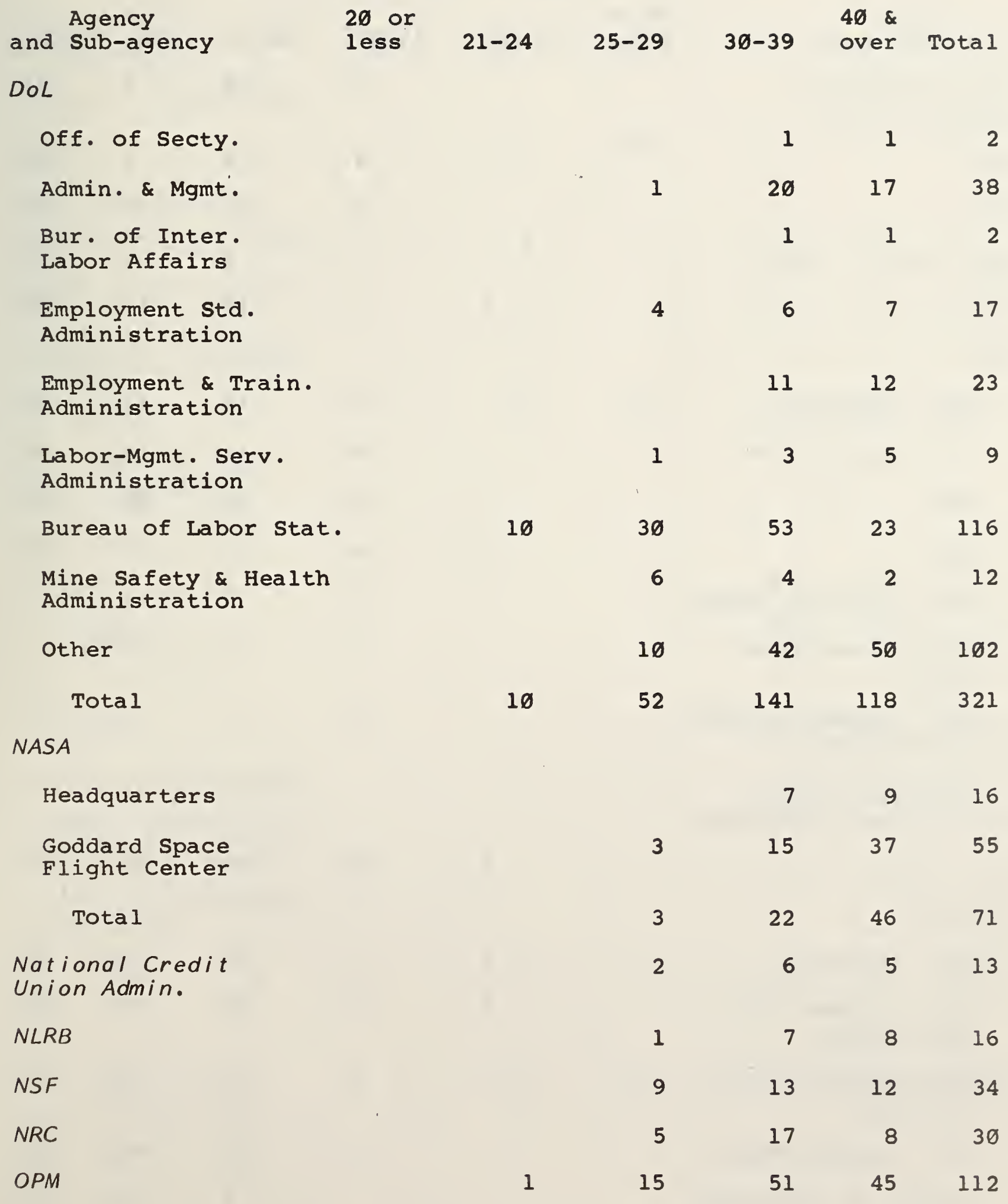


TABLE 6. (continued)

Age Summary

Agency
and Sub-agency
Pension Benefit
Guaranty Corp.
SEC
SBA

20 or

less $21-24$ (1)

25-29

5

30-39

$40 \&$

over Total

1

4

18

8

31

14

9

28

Smithsonian Instit.

State

6

2

DOT

Off. of Secty.
U.S. Coast Guard
FAA

FHA

1

4

Fed. Railroad Admin.

Urban Mass Transp.

Admin.

Nat. Highway Traffic

Safety Admin.

Research \& Spec.

Programs Directorate

$$
\text { Total }
$$

5

35

1

7

3

4

5

19

1

1

2

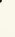

12

30

44

1

.

9

9

19

14

11

38

14

15

15

33

21

19

44

28

40

74

21

10

54

1

7

1

9

3

3

7

1

3

6

2

1

3

98

92

230

Treasury

Off. of secty.

1

7

34

14

56

Bur. of Govt. Fin.

3

8

59

37

107

Operations

Comptroller of the Currency

U.S. Customs Serv.

1

3

1

33

49

83

6

6

12 
TABLE 6 (continued)

Age Summary

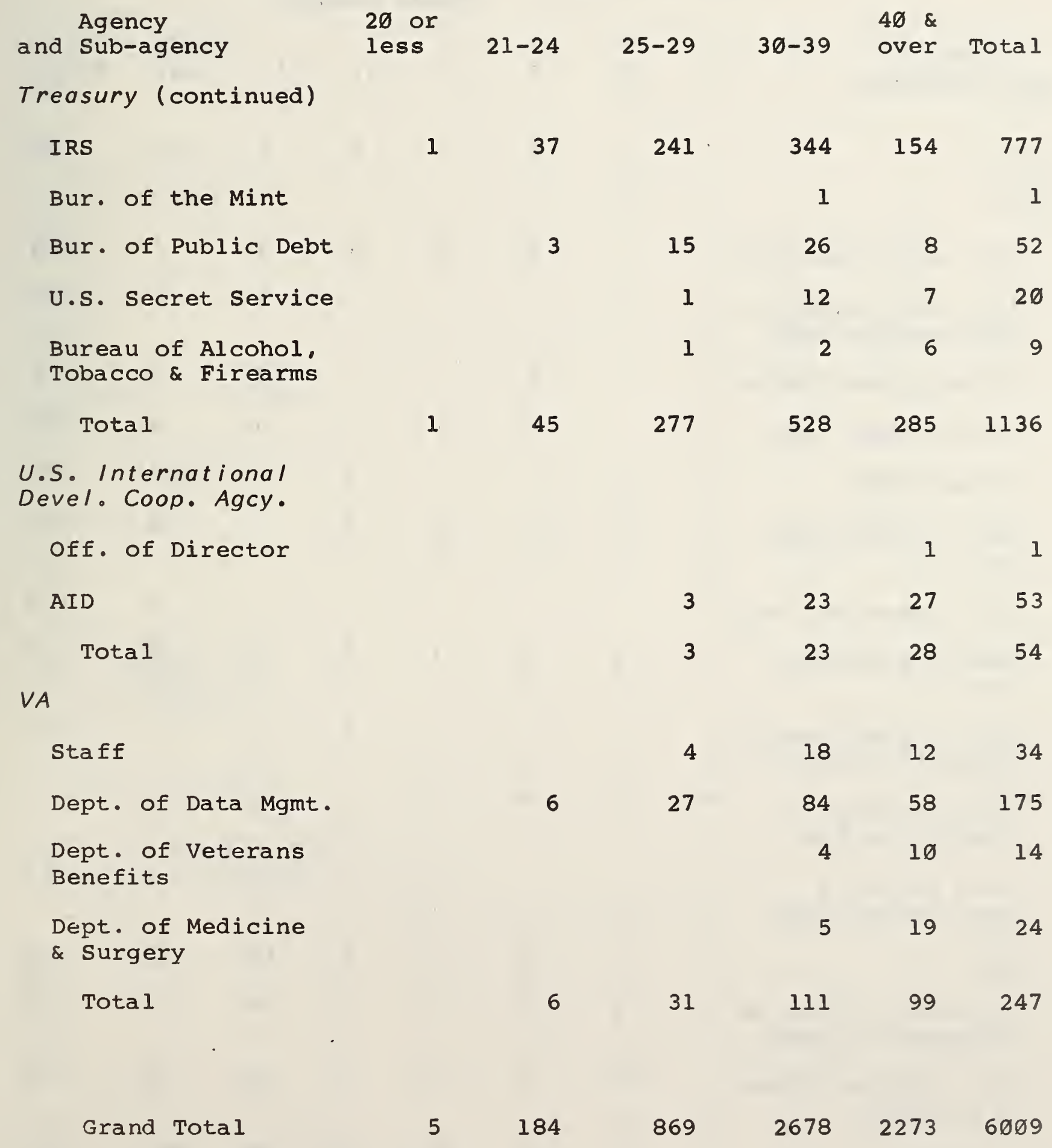


TABLE 7. Number of Computer Specialists by Grade Levels for Agencies and Sub-agencies.

\section{Grade summary}

Agency

and sub-agency

ACTION

USDA

Agr. Marketing Serv.

Agr. Stabilization

Conservation Serv.

Farmers Home Admin.

Foreign Agr. Serv.

Forest Serv.

Soil Conservation

Serv.

Off. Inspector Gen.

Food \& Nutrition

serv.

Anm. \& Plant Health

Inspection Serv.

Federal Grain

Inspection Serv.

Food Safety \&

Quality Service

\section{SEA}

Economics, Stat. \&

Cooperative Serv.

Off. of Operations

\& Finance

Tota 1 $\begin{array}{lllllll}13 \& & 9 & 11 & \text { over } & \text { Total }\end{array}$

12

6

7

11

27

3

3

4

3

1

14

1

6

7

1

2

6

9

1

3

1

1

3

1

2

5

9

18

6

28

37

8

15

4

4

1

2

3

5

9

20

40

3

2

1

2

4

12

1

1

1

8

3

5

$9 \quad 14$

23

54

1

6

9

819

27

70

1

1

3

215

38

60

5

23

30

34

85

181

358 
TABLE 7. (continued)

$$
\text { Grade summary }
$$

\begin{tabular}{|c|c|c|c|c|c|c|c|}
\hline $\begin{array}{l}\text { Agency } \\
\text { and Sub-agency }\end{array}$ & 5 & 7 & 9 & 11 & 12 & $\begin{array}{l}13 \& \\
\text { over }\end{array}$ & Total \\
\hline$C A B$ & & & 1 & 4 & 2 & 17 & 24 \\
\hline \multicolumn{8}{|l|}{ DoC } \\
\hline Off. of Secty. & & & 3 & 6 & 3 & 29 & 41 \\
\hline EDA & 1 & 3 & 1 & 3 & 5 & 8 & 21 \\
\hline BEA & & 1 & 5 & 3 & 6 & 7 & 22 \\
\hline NOAA & 5 & 13 & 15 & 32 & 64 & 108 & 237 \\
\hline $\begin{array}{l}\text { Internat. \& Trade } \\
\text { Admin. }\end{array}$ & & & 1 & 1 & 2 & 2 & 6 \\
\hline Maritime Admin. & & 4 & 2 & 2 & 6 & 23 & 37 \\
\hline Patent off. & 3 & 1 & 2 & 3 & 18 & 19 & 46 \\
\hline NBS & 2 & 6 & 8 & $1 \varnothing$ & 14 & 35 & 75 \\
\hline MBD & & & & & & 3 & 3 \\
\hline $\begin{array}{l}\text { Nat. Telecom. \& } \\
\text { Infor. Agency }\end{array}$ & f & & & & 3 & 4 & 7 \\
\hline NTIS & & & 1 & 2 & 9 & 8 & $2 \varnothing$ \\
\hline Bureau of Census & 10 & 35 & 44 & 54 & 122 & 163 & 428 \\
\hline $\begin{array}{l}\text { Off. of Federal stat. } \\
\text { Policy \& Standards }\end{array}$ & & & & & & 1 & 1 \\
\hline Total & 21 & 63 & 82 & 116 & 252 & 410 & 944 \\
\hline CSA & & 2 & 3 & 6 & 8 & 11 & 30 \\
\hline CPSC & & 1 & & 1 & 7 & 7 & 16 \\
\hline$E P A$ & 1 & 9 & 10 & 9 & 11 & $4 \varnothing$ & $8 \emptyset$ \\
\hline$D O E$ & & 3 & 5 & 19 & 26 & 144 & 197 \\
\hline FCC & 5 & 3 & 7 & 4 & 23 & 17 & 59 \\
\hline$F D / C$ & 2 & 6 & 5 & 4 & 6 & 32 & 55 \\
\hline
\end{tabular}


TABLE 7. (continued)

\section{Grade Surmary}

\author{
Agency \\ and Sub-agency \\ Federal Energy \\ Management Agency
}

FHLB

FTC

GSA

Off. of Preparedness

Public Bldg. Serv.

Fed. Supply Serv.

National Archives \&

Records Serv.

Automated Data \&

Telecomm. Serv.

Transport. \& Public

Utilities Serv.

Off. of Admin.

Off. of Inspector

General

Total

HHS

Off. of Secty.

Off. of Asst.

Secty. for Health

Alcohol, Drug Abuse

\& Mental Health

Health Serv.

Administration $\begin{array}{lllllll}13 \& & 1\end{array} \quad 9 \quad 11 \quad \begin{aligned} & \text { over } \\ & \text { Total }\end{aligned}$

$\begin{array}{llllll}1 & 1 & 6 & 6 & 11 & 25\end{array}$

23

$\begin{array}{llll}5 & 8 & 14 & 32\end{array}$

31

$8 \quad 10 \quad 22$

$8 \quad 10 \quad 22$

$2 \quad 2$

4

14

4210

9

$3 \varnothing$

11

2

11

1

3

$1 \quad 1,20$

33

55

1

3

4

$2 \quad 12$

15

20

66

35

150

1

1

316

21

$27 \quad 101$

81

249

$$
2
$$

$$
2
$$

9

13

22

69

117

1

1

1

$4 \quad 14$

21

42

1

2

3

6

6

14

32

1

1

1

1

4

15

FDA 
TABLE 7. (continued)

Grade Summary

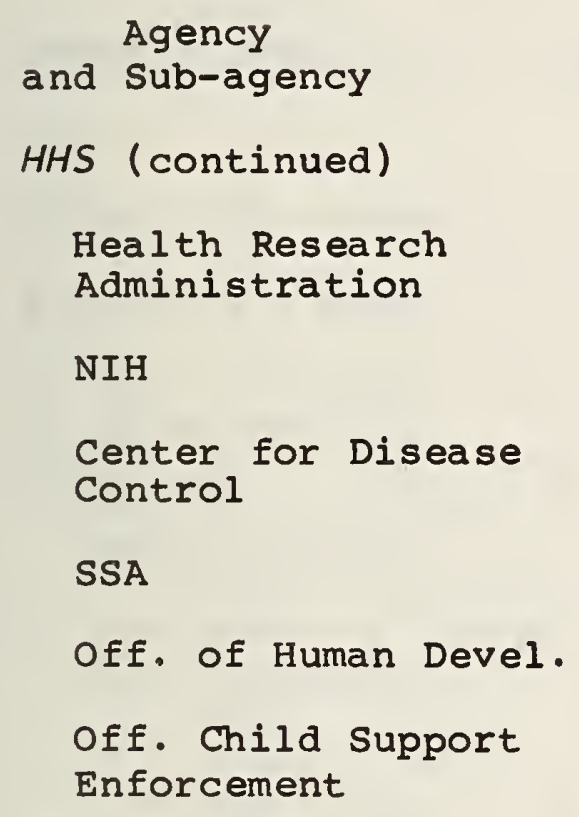

5

11

4

5

7

$1 \varnothing$

28

$\begin{array}{llll}7 & 17 & 29 & 36\end{array}$

$84 \quad 112$

285

1

1

2

1

17

11

31

2

2

6

10

14

6

11

$16 \quad 33$

63

$82 \quad 199$

330

723

1 3

211

27

128

172

1

2

1

1

2

4

1

3

2

12

29

140 
TABLE 7. (continued)

Grade Summary

Agency

and Sub-agency

5

Dol (continued)

Geological Survey

6

$6 \quad 11 \quad 30$

56

109

Bureau of Mines

National Park Ser.

U.S. Fish \& Wildlife

Service

Heritage Conservation

\& Recreation Service

Off. of Surface Mining,

Reclamation \& Enforce.

Total

6

2

6

7

15

1

6

5

12

13

4

6

14

2

1

3

6

2

2

Internat. Commun.

Agency

ICC

1

Justice

Offices, Div. \&

Boards

FBI

5

6

$9 \quad 10 \quad 18$

62

105

Bur. of Prisons

Immigration \&

1

7

4

$1 \varnothing$

23

34

83

Naturalization Serv.

Bur. of Prisons

Industries

Drug Enforcement

Administration

Off. of Justice

Assistance

U.S. Marshals Serv.

Total

1

$1 \quad 12$

14

31

61

3

5

$2 \varnothing$

36

1

3

4

1

2

2

2

2

28

37

1

$1 \varnothing$

11

11

2

1

5 
TABLE 7. (continued)

Grade Summary

\begin{tabular}{|c|c|c|c|c|c|c|c|}
\hline $\begin{array}{c}\text { Agency } \\
\text { and Sub-agency }\end{array}$ & 5 & 7 & 9 & 11 & 12 & $\begin{array}{l}13 \& \\
\text { over }\end{array}$ & Total \\
\hline \multicolumn{8}{|l|}{ DoL } \\
\hline Off. of Secty. & & & & & 1 & 1 & 2 \\
\hline Admin. \& Mgmt. & & & 3 & 4 & 16 & 15 & 38 \\
\hline $\begin{array}{l}\text { Bur. of Inter. } \\
\text { Labor Affairs }\end{array}$ & & & & & 1 & 1 & 2 \\
\hline $\begin{array}{l}\text { Employment Stds. } \\
\text { Administration }\end{array}$ & 1 & 1 & 2 & 2 & 4 & 8 & 18 \\
\hline $\begin{array}{l}\text { Employment \& Train. } \\
\text { Administration }\end{array}$ & & & & 6 & 7 & 10 & 23 \\
\hline $\begin{array}{l}\text { Labor-Mgmt. Serv. } \\
\text { Administration }\end{array}$ & & & & 2 & 3 & 4 & 9 \\
\hline Bureau of Labor Stat. & 5 & 23 & $2 \varnothing$ & 18 & 33 & $2 \varnothing$ & 119 \\
\hline $\begin{array}{l}\text { Mine Safety \& Health } \\
\text { Administration }\end{array}$ & & 1 & 1 & 3 & 5 & 2 & 12 \\
\hline Other & 5 & 9 & 5 & 13 & 26 & 44 & $1 \varnothing 2$ \\
\hline Total & 11 & 34 & 31 & 48 & 96 & $1 ø 5$ & 325 \\
\hline
\end{tabular}

NASA

Headquarters

Goddard Space

Flight Center

11

3

2

13

16

Total

1

1

3

$6 \quad 26$

34

71

National Credit

1

2

Union Administration

$N L R B$

2

4

2

4

16

NSF

2

NRC

1

5

2

1

22

1

2

3

5

$2 \emptyset$ 
TABLE 7. (continued)

Grade Summary

\begin{tabular}{|c|c|c|c|c|c|c|c|}
\hline $\begin{array}{c}\text { Agency } \\
\text { and Sub-agency }\end{array}$ & 5 & 7 & 9 & 11 & 12 & $\begin{array}{l}13 \& \\
\text { over }\end{array}$ & Total \\
\hline$O P M$ & 1 & 14 & 8 & 11 & 36 & 26 & 96 \\
\hline $\begin{array}{l}\text { Pension Benefit } \\
\text { Guaranty Corp. }\end{array}$ & 2 & 1 & 3 & 4 & 6 & 15 & 31 \\
\hline SEC & 2 & 2 & 5 & & 9 & 11 & 29 \\
\hline$S B A$ & 1 & 1 & 1 & 1 & 9 & 1 & 14 \\
\hline Smithsonian Instit. & & & 1 & 4 & 1 & 13 & 19 \\
\hline State & 7 & 2 & 4 & 3 & 8 & 13 & 37 \\
\hline \multicolumn{8}{|l|}{ DOT } \\
\hline Off. of Secty. & & 2 & 2 & & 4 & 25 & 33 \\
\hline U.S. Coast Guard & 1 & 3 & 11 & 4 & 8 & 17 & 44 \\
\hline FAA & & 2 & 4 & 11 & 9 & 48 & 74 \\
\hline FHA & 13 & & 6 & 5 & 17 & 12 & 53 \\
\hline Fed. Railroad Admin. & 1 & & 1 & 1 & 1 & 5 & 9 \\
\hline $\begin{array}{l}\text { Urban Mass Transp. } \\
\text { Admin. }\end{array}$ & & & 2 & & 2 & 3 & 7 \\
\hline $\begin{array}{l}\text { Nat. Highway Traffic } \\
\text { Safety Admin. }\end{array}$ & 1 & 2 & 1 & & & 2 & 6 \\
\hline $\begin{array}{l}\text { Research \& Spec. } \\
\text { Programs Directorate }\end{array}$ & & & 1 & & 1 & 1 & 3 \\
\hline Total & 16 & 9 & 28 & 21 & 42 & 113 & 229 \\
\hline \multicolumn{8}{|l|}{ Treasury } \\
\hline off. of Secty. & & & 2 & 8 & 17 & 29 & 56 \\
\hline $\begin{array}{l}\text { Bur. of Govt. Fin. } \\
\text { Operations }\end{array}$ & 4 & 3 & 12 & 9 & 31 & 48 & 107 \\
\hline $\begin{array}{l}\text { Comptroller of the } \\
\text { Currency }\end{array}$ & 1 & 2 & & 3 & 7 & 7 & $2 \varnothing$ \\
\hline U.S. Customs Serv. & & & 1 & 4 & 21 & 57 & 83 \\
\hline
\end{tabular}


TABLE 7. (continued)

Grade Summary

\begin{tabular}{|c|c|c|c|c|c|c|c|}
\hline $\begin{array}{l}\text { Agency } \\
\text { and Sub-agency }\end{array}$ & 5 & 7 & 9 & 11 & 12 & $\begin{array}{l}13 \& \\
\text { over }\end{array}$ & Total \\
\hline \multicolumn{8}{|l|}{ Treasury (continued) } \\
\hline $\begin{array}{l}\text { Bureau of Engraving } \\
\text { \& Printing }\end{array}$ & & & & 5 & 3 & 4 & 12 \\
\hline IRS & 102 & 135 & 77 & 46 & 175 & 249 & 782 \\
\hline Bur. of the Mint & & & & 1 & & & 1 \\
\hline Bur. of Public Debt & 5 & 10 & 5 & 4 & 19 & 9 & 52 \\
\hline U.S. Secret Service & 1 & & 4 & 4 & 2 & 9 & 20 \\
\hline $\begin{array}{l}\text { Bureau of Alcohol, } \\
\text { Tobacco \& Firearms }\end{array}$ & & 1 & & & 1 & 7 & 9 \\
\hline Total & 113 & 151 & 101 & 84 & 276 & 419 & 1144 \\
\hline \multicolumn{8}{|l|}{$\begin{array}{l}\text { U.S. International } \\
\text { Devel. Coop. Agcy. }\end{array}$} \\
\hline Off. of Director & & & & & 1 & & 1 \\
\hline AID & & & 2 & 1 & 17 & 33 & 53 \\
\hline Total & & & 2 & 1 & 18 & 33 & 54 \\
\hline \multicolumn{8}{|l|}{$V A$} \\
\hline Stäff & & 2 & 3 & 1 & 7 & 22 & 35 \\
\hline Dept. of Data Mgmt. & 9 & 19 & 12 & 9 & 41 & 86 & 176 \\
\hline $\begin{array}{l}\text { Dept. of Veterans } \\
\text { Benefits }\end{array}$ & & & & 1 & 1 & 12 & 14 \\
\hline $\begin{array}{l}\text { Dept of Medicine } \\
\& \text { Surgery }\end{array}$ & & & 1 & & 4 & 19 & 24 \\
\hline Total & 9 & 21 & 16 & 11 & 53 & 139 & 249 \\
\hline Grand Total & $23 \varnothing$ & 440 & 473 & 591 & 1494 & 2718 & 5946 \\
\hline
\end{tabular}


TABLE 8. Number of Computer Specialists by Educational Levels for Agencies and Sub-agencies.

Education Summary

Agency
and Sub-agency

ACTION

USDA

Agr. Marketing Serv.

Agr. Stabilization \&

Conservation Serv.

Farmers Home Admin.

Foreign Agr. Serv.

Forest Service

Soil Conservation

Serv.

Off. Inspector Gen.

Food \& Nutrition Serv.

Anm. \& Plant Health

Inspection Serv.

Federal Grain

Inspection Serv.

Food Safety \&

Quality Service

SEA

Economics, stat. \&

Cooperative Serv.

Off. of Operations

\& Finance

Tota 1

$C A B$

DoC
High

School

18

6

2

2

7

14

7

1

24

8

12

29

33

21

166

16
B.A.

8

College Degree

M.A.

PhD.

Tota 1

1

27

14

7

9

18

37

15

4

40

12

1

17

13

12

1

55

30

6

69

26

11

2

60

143

46

3

358

7

1

24 
TABLE 8. (continued)

\begin{tabular}{|c|c|c|c|c|c|}
\hline Agency & High & & ge Dec & & \\
\hline and Sub-agency & School & B.A. & M.A. & PhD. & Total \\
\hline DoC (continuted) & & & & & \\
\hline EDA & 10 & 9 & 2 & & 21 \\
\hline BEA & 10 & 9 & 3 & & 22 \\
\hline NOAA & 113 & 93 & 24 & 3 & 233 \\
\hline $\begin{array}{l}\text { Internat. \& Trade } \\
\text { Admin. }\end{array}$ & 2 & 3 & & & 5 \\
\hline Maritime Admin. & 24 & 5 & 2 & & 31 \\
\hline Patent off. & 30 & 14 & 2 & & 46 \\
\hline NBS & 33 & 30 & 10 & 2 & 75 \\
\hline MBD & 1 & 2 & & & 3 \\
\hline $\begin{array}{l}\text { Nat. Telecom. \& } \\
\text { Infor. Agency }\end{array}$ & 6 & 1 & & & 7 \\
\hline NTIS & 14 & 6 & & & 20 \\
\hline Bureau of Census & 121 & 251 & 44 & 4 & 420 \\
\hline $\begin{array}{l}\text { Off. of Federal Stat. } \\
\text { Policy \& Standards }\end{array}$ & 1 & & 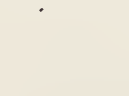 & & 1 \\
\hline Total & 389 & 435 & 91 & 9 & 924 \\
\hline CSA & 23 & 7 & & & 30 \\
\hline CPSC & 8 & 7 & 1 & & 16 \\
\hline$E P A$ & 34 & 32 & 16 & 1 & 83 \\
\hline$D O E$ & 106 & 62 & 26 & 4 & 198 \\
\hline$F C C$ & 31 & 25 & 3 & & 59 \\
\hline$F D / C$ & 31 & 22 & 5 & & 58 \\
\hline $\begin{array}{l}\text { Federal Energy } \\
\text { Management Agency }\end{array}$ & 16 & 8 & 1 & & 25 \\
\hline$F H L B$ & 17 & 12 & 2 & 1 & 32 \\
\hline
\end{tabular}


TABLE 8. (continued)

Education Summary

Agency
and Sub-agency

FTC

GSA

Off. of Preparedness

Public Bldg. Serv.

Fed. Supply serv.

National Archives \&

Records Serv.

Automated Data \&

Telecomm. Serv.

Transport \& Public

Utilities Serv.

Off. of Admin.

Off. of Inspector

General

Total
High

School

6

3

16

1

3

25

2

$10 \varnothing$

$15 \emptyset$

70

20

Off. of Asst.

Secty. for Health

Alcohol, Drug Abuse

\& Mental Health

Health Serv.

Administration

FDA

Health Research

Administration

NIH 
TABLE 8. (continued)

\section{Agency \\ and Sub-agency \\ HHS (continued)}

Off. of Human Devel.

Off. of Child support

Enforcement

Total

HUD

Asst. secty. for

Administration

Asst. Secty. for

Comm. Plan. Devel.

Fair Housing \&

Equal Opportunity

Asst. Secty. for

Housing

Other

Total

Dol

Off. of secty.

Bur. of Land Mgmt.

Bur. of Indian Affairs

Water \& Power Resources

Geological Survey

Bureau of Mines

National Park Serv.

U.S. Fish \& Wildlife

Service

343

High

School

9

3

76

48

13

2

2

1

1

2

3

1

6

2

80

1

53

16

149

2

1

3

1

1

1

38

51

13

2

104

9

6

15

5

6

1

12

4

30

10

10

04

6

6

4 
TABLE 8. (continued)

Education Summary

\section{Agency}

and Sub-agency

Dol (continued)

Heritage Conservation

\& Recreation Service

Off. of Surface Mining

Reclamation \& Enforce.

Total

Agency

ICC

Offices, Div . \&

Boards

FBI

Bur. of Prisons

Immigration \&

Naturalization Serv.

Bur. of Prisons

Industries

Drug Enforcement

Administration

Off. of Justice

Assistance

U.S. Marshals Ser.

Total
4

64

15

11

High
school

1

12

4

27

73

$2 \varnothing$

2

159

23
49

$4 \varnothing$

14

6

2

44

8

103

36

6

1

83

31

35

4

37

22

9

6

12

4

5

3

1

5

156

119

32

3

310

DoL

Off. of Secty.

Admin. \& Mgmt. 
TABLE 8. (continued)

\section{Education Summary}

Agency

and Sub-agency

DoL (continued)

Employment Std.

Admin.

Employment \& Train.

Administration

Labor-Mgmt. Serv .

Admin.

Bur. of Labor stat.

Mine Safety \& Health

Administration

Other

Total

NASA

Headquarters

Goddard Space

Flight Center

Total

National Credit

Union Admin.

$N L R B$

NSF

NRC

$O P M$

Pension Benefit

Guaranty Corp.
High

School

9

11

7

46

6

47

156

3

39

42

2

10

14

12

60

17

15
College Degree

B.A. M.A. PhD. Total

7

1

1

18

19

9

59

12

1

118

4

10

36

13

96

124

27

2

309

16

55

71

13

15

32

28

112

31

29

30 
TABLE 8. (continued)

Education Summary

High School

3

14

21

21

53

10

FHA

Fed. Railroad Admin.

Urban Mass Transp.

Admin.

Nat. Highway Traffic Safety Admin.

Research \& Special

Programs Directorate

Tota 1

116

6

3

1

1

26

56

Bur. of Govt. Fin.

Operations

Comptroller of the

Currency

U.S. Customs Serv.

Bureau of Engraving

\& Printing

IRS

321

Bur. of the Mint

10

47

10

20

10

56

43

3

102

6

29

2

437

29

1

788

Bur. of Public Debt 
TABLE 8. (continued)

$$
\begin{aligned}
& \text { Agency } \\
& \text { and Sub-agency } \\
& \text { Treasury (continued) } \\
& \text { U.S. Secret Serv. } \\
& \text { Bur. of Alcohol, } \\
& \text { Tobacco \& Firearms }
\end{aligned}
$$$$
\text { Tota } 1
$$$$
\text { U.S. International }
$$$$
\text { Develop. Coop. Agency }
$$$$
\text { off. of Director }
$$$$
\text { AID }
$$$$
\text { Total }
$$

$V A$

$$
\begin{aligned}
& \text { Staff } \\
& \text { Dept. of Data Mgmt. } \\
& \text { Dept. of Veterans } \\
& \text { Benefits } \\
& \text { Dept. of Medicine } \\
& \text { \& Surgery }
\end{aligned}
$$$$
\text { Tota } 1
$$

Grand Total
High School 

COMPUTER PROGRAMMER SURVEY

\section{INDIVIDUAL PROGRAMMER PROFILE}

1. Job title (including code):

2. Age:
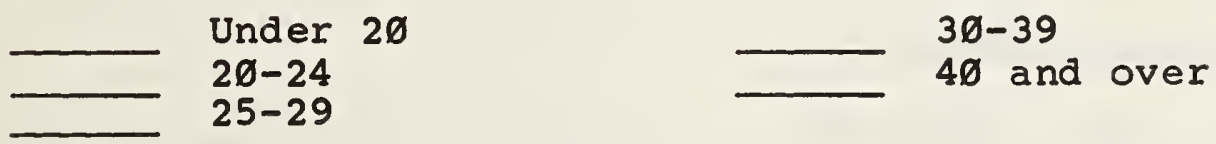

3. GS grade equivalent:
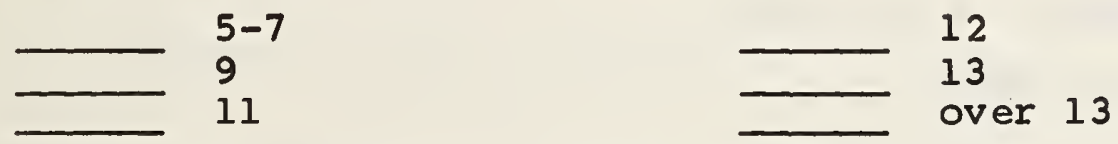

4. Time in present job (current agency):
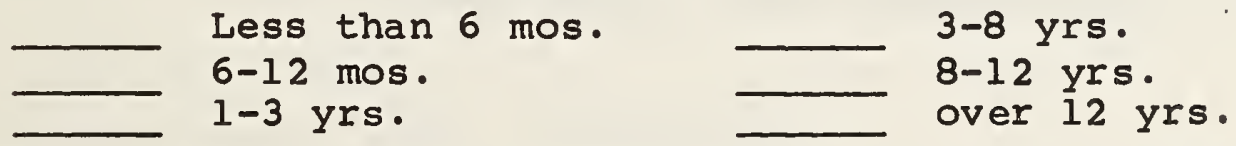

5. Education and training:

a. Highest general academic level:

\section{High school}

- Bachelor's degree Master's degree Doctorate (PhD)

b. Highest academic level with ADP major:

Degree

c. Other (identify and give length of training) types of training (include in-house): 
6. Programming languages used:

a. Most frequently

b. Others used in past year

7. Computer system used:

a. Most frequently

b. Percent work done:

1) Batch

2) Interactively

c. List systems used in past year

8. Types of activities: (by time spent; e.g. $\emptyset$ - none, 1 - most 2 - next, etc.)
a. design
b. code
c. test
d. document

e. contract monitor

f. maintenance

g. other (list)

9. Type of projects over the past year: (percent of time)

\section{Applications Systems}

Programs Programs Other
a. New development
b. Conversion of old programs to new systems
c. Creation of new versions of old programs
d. Maintenance of existing systems 
Note: State what other is:

10. Type of applications program: (briefly describe and indicate of time spent on each)

a. Business data processing

b. Information processing

c. Scientific data processing

d. Other (1ist)

11. Do you manage other programmers? No Yes Number 



\section{COMPUTER PROGRAMMER SURVEY \\ DESCRIPTION OF ACTIVITY OF ORGANIZATION}

If additional space is required, use a separate sheet of paper identifying the appropriate items.

A. Name of computer organization (the identity of survey participants will be kept confidential).

B. Internal organizational structure and number of employees in each unit:

C. Inventory of computer hardware:

D. List of software functional activities:

E. Levels of effort in project development in man-years (choose a typical project)

1. Requirements analysis

2. Functional requirements/specifications

3. Design

4. Development

5. Operations

6. Evaluation 
F. Programming activities:

1. Type: (show size of staff or man-years \& briefly describe work)

a. Systems

b. Applications

1. Business

2. Scientific

3. Other (specify)

c. Other (specify)

2. Phase of programming: (estimate $\&$ of time in each area for organization)

a. New development

b. Conversion

c. Maintenance

d. Other

3. Programming languages used: (estimate 8 of each for organization)

a. Principal

b. Other 
4. List computer systems operated and estimate 8 of programming accomplished for each system:

5. Description of contract support:

a. Estimate total volume of contract support (man-years \& dollars)

b. Estimate $\&$ of total programming done by contract (where appropriate, make estimate by type of programming)

c. General descriptive information re contract management, including quality control, insurance of timely performance, etc.

6. Programmer recruiting:

a. Sources

b. Means of contact

c. Special programs

d. General description, including special problems and major difficulties 
G. Quality control for in-house software:

1. Standards used (languages and methodologies; FIPS, internal or other)

2. Guidelines used (languages and methodologies; internal or other)

3. Tools used (e.g. design, test coverage analysis, editors) - give name of tool

4. Tools to which you have access but don't use - state reason it is not used, e.g., not helpful, use too much machine time, etc.

5. Is there a procedure for Quality Assurance comprehensive moderate none

6. Is there a procedure for testing (new and maintenance) comprehensive moderate none

7. Is training given in "best" methods extensive some none in-house outside 
8. If standards are used, are they: standard excellent good adequate bad

9. Area(s) in which software quality improvement would make the most difference, e.g., tools, training, standard guidelines

1ø. What type of guidance would you like and in what areas - order by preference

11. If your needs are not covered above - use the space below to list them. 
NBS-114A (REV. 2.80)

U.S. DEPT. OF COMM.

BIBLIOGRAPHIC DATA

SHEET (See in structions)

1. PUBLICATION OR REPORT NO.

NBSIR 82-2565
2. Performing Organ. Report Nod 3. Publication Date

March 1983

4. TITLE AND SUBTITLE

Profiles of Computer Rrogrammers in the Executive Branch of the Federal Government

\section{AUTHOR(S)}

Patricia B. Powel1, ed.

6. PERFORMING ORGANIZATION (If joint or other than NBS, see instructions)

NATIONAL BUREAU OF STANDAROS

Ralph A. Simmons

DEPARTMENT OF COMMERCE

WASHINGTON, D.C. 20234

Independent Contractor

7. Contract Grant No.

NB800NAA4467

8. Type of Report \& Period Covered

Final

9. SPONSORING ORGANIZATION NAME AND COMPLETE ADDRESS (Street, City, StOte, ZIF)

Patricia B. Powell

National Bureau of Standards

B1 dg. 225, Room B266

Washington, DC 20234

10. SUPPLEMENTARY NOTES

Document describes a computer program; SF-185, FIPS Software Summary, is attached.

11. ABSTRACT (A 200-word or less factual summary of most significant information. If document includes a significant bibliography or literature survey, mention it here)

The report is a detailed programmer survey compiled from interviews with eight selected organizations and an OPM data base. The survey includes staffing, hardware, programming activities and languages, contract support, programmer recruiting, quality control, personnel profile, and programmer activities. The OPM data base is summarized by age, grade and education for Computer Specialists in the Washington Metropolitan Area.

12. KEY WORDS (Six to twelve entries; alphabetical order; capitalize only proper names; and separate key words by semicolons) computer programmers; computer specialist; Federal civilian organizations; OPM data base; profile of computer programmers

13. AVAILABILITY

Unlimited

For Official Distribution. Do Not Release to NTIS

Order From Superintendent of Documents, U.S. Government Printing Office, Washington, D.C. 20402.

14. NO. OF

PRINTED PAGES

81

Order From National Technical Information Service (NTIS), Springfield, VA. 22161

15. Price

$\$ 11.50$ 

